\title{
STABILITY OF DYNAMIC RESPONSE OF SUSPENSION BRIDGES
}

\author{
Antonio Capsoni ${ }^{*}$, Raffaele Ardito ${ }^{2}$, and Andrea Guerrieri ${ }^{2}$ \\ ${ }^{1}$ Dept. of Architecture, Built Environment and Construction Engineering, Politecnico di Milano \\ Piazza Leonardo da Vinci 32, Milan, Italy \\ ${ }^{2}$ Dept. of Civil and Environmental Engineering, Politecnico di Milano \\ Piazza Leonardo da Vinci 32, Milan, Italy
}

Keywords: Suspension Bridges, Non-linear Dynamics, Aeroelasticity, Stability, Parametric Resonance, Floquet Theory.

\begin{abstract}
The potential occurrence of internal parametric resonance phenomena has been recently indicated as a potential contributory cause of the appearance of critical dynamic states in long-span suspension bridges. At the same time, suspension bridges, in view of their flexibility, are prone to aeroelastic response, such as vortex shedding, torsional divergence and flutter. In this paper, a non-linear dynamic model of a suspension bridge is devised, with the purpose of providing a first attempt toward a unified framework for the study of aeroelastic and internal resonance instabilities. Inspired by the pioneering work of Herrmann and Hauger, the analyses have been based on a linearized formulation that is able to represent the main structural non-linear effects and the coupling given by aerodynamic forces. The results confirm that the interaction between aeroelastic effects and non-linear internal resonance leads to unstable conditions for wind speeds which can be lower than the critical threshold for standard aeroelastic predictions.
\end{abstract}

\section{INTRODUCTION}

Suspension bridges represent a spontaneous answer to demands of large spans, lightweight, high strength, ease of construction and aesthetic appearance. On one hand, the flexibility caused by the cable system and by the long span make the suspension bridges sensitive to dynamic loads; on the other hand, the relatively simple geometry of cable structures makes continuum approaches still very attractive, since can be based on a minimal number of non-dimensional parameters.

Early attempts to address the static equilibrium of suspension bridges were made by Moisseiff, who extended the elastic theory to the well-established Deflection Theory [1,2] by enforcing equilibrium in the deformed position, and accounting for the stiffening effect in the main cables. Earliest continuum models for the linear vertical vibrations of suspension bridges reproduced the effects of the stiffening truss girder by means of a Euler-Bernoulli beam supported by the main cables through inextensible and distributed vertical hangers. In this regard, the classic continuum model for the linear vertical vibration of suspension bridges, based on the so called linearized deflection theory, was first proposed by Bleich et al. [3], and Steinman [4], who

*Corresponding Author. E-mail address: antonio.capsoni@polimi.it 
derived some formulas for computing natural frequencies and mode shapes, and recently reviewed by Luco and Turmo [5]. The latter authors showed that the linear vibration of the considered suspension bridge model is completely governed by two non-dimensional parameters: the classic Irvine parameter of suspended cables, first introduced by Irvine [6], and a second parameter accounting for the relative stiffness of the girder with respect to the main cable system. Abdel-Ghaffar in the late 1970's [7-9] developed the methodology of free vertical, torsional and lateral vibration analysis of suspension bridges by means of a variational principle and a finite element approach. Then, the same author [10-12] extended the continuum formulation to include coupling between vertical-torsional vibrations, nonlinear effects occurring in the case of large vibrations and the effects of distortional deformation of the girder cross-section. Nowadays, in the design of suspension bridges a comprehensive set of wind related responses are taken into consideration, such as static divergence, vortex-shedding, buffeting and flutter. Indeed, the risk of developing aeroelastic instabilities is always present in lightweight longspan structures, characterized by high flexibility, low bending-to-torsional stiffness ratio and high width-to-depth ratio. Although such phenomena were already well known in aviation, aeroelastic effects did not represent an important issue in bridge design before the collapse of the Tacoma Narrows Bridge (USA) in 1940. Such a catastrophe was seen mainly as a direct consequence of flutter [13] that developed on the bridge deck at wind speed much lower than the design one. Flutter is generally studied within linearized aeroelastic models, which can provide the range of wind speeds where Hopf bifurcation occurs. To consider the effects due to the unsteadiness of the relative motion between the section and the air flow, indicial Theodorsen type [14-16] formulations can be adopted to predict more accurately the critical wind speed at the onset of the flutter instability [17] with respect to the quasi-steady formulation. The equations of motion for suspension bridges were employed for aeroelastic investigations in [18], where analysis were centered on experimentally determined flutter derivatives, and a full threedimensional modal analysis of the structure.

It is well known from non-linear dynamics that, between coupled oscillators, energy transfer $[19,20]$ can occur as far as the energetic levels reaches well-established critical thresholds. Classically, this behaviour is referred to as the internal resonance phenomenon. Many authors applied these principles to study the vibrations response of suspension bridges. The authors of $[21,22]$ used the continuous model proposed by Abdel-Ghaffar [11], and solved the system of equations by means of the multiple scale perturbative technique [23]. Recently, Arioli and Gazzola [24], trying to explain why torsional oscillations suddenly appeared before the Tacoma Narrows collapse, found out that, also in isolated systems, vertical oscillations may switch to torsional ones, as long as they become large enough. The problem was already tackled by other authors [25-29] but it seems that there is still an open issue regarding the complete explanation of the sudden appearance of large oscillations which led to collapse. Hence, they paved the way for future works concerning the interaction between internal resonance and aeroelastic phenomena, as the present paper wants to do.

Indeed, the present article intends to study the stability of a suspension bridge model, following the preliminary attempt described in [27]. The analysis will exploit the continuum formulation of Abdel-Ghaffar [11], enriched by the aeroelastic actions coming from Theodorsen [16] indicial formulation for the wind-structure interaction. The stability will be checked in Lyapunov asymptotic sense, exploiting the well-known Floquet theory [30]. The variational system of equations is obtained following the pioneering procedure proposed by Herrman and Hauger [31], who assumed small but finite flexural perturbations coming from vortex-shedding excitation. The possibility of parametric internal resonances such as harmonic, sub-harmonic and super-harmonic, or additive combinational and anti-resonances will be checked by means of suitable stability maps. 


\section{THE STRUCTURAL MODEL}

A continuum model of single span, linearly elastic, suspension bridge is considered (Fig. 1). The bridge, having a span length $l$, is composed of two main cables that support the stiffening girder (bridge deck) through uniformly distributed, massless and inextensible vertical hangers. The main cables are hinged at fixed anchors placed at the same vertical elevation and are modeled as mono-dimensional elements with negligible flexural, torsional and shear rigidities. The stiffening girder is modeled as an equivalent, uniform, Euler-Bernoulli beam, with flexural hinges and torsional forks at its ends. The distortional deformation of the cross-section is neglected. The cross-section of the girder is symmetric with respect to vertical local axis $y$ (Fig. 1). The contribution of the stiffening girder in carrying dead loads is disregarded: dead loads are entirely carried by the main cables and are assumed to be uniformly distributed along the longitudinal axis.

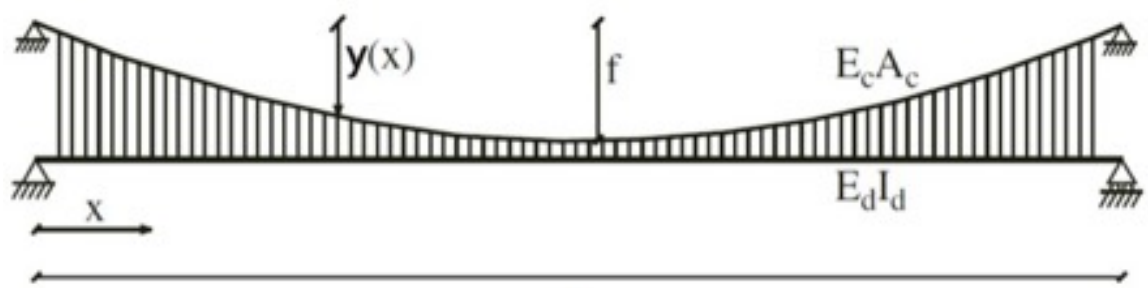

$\mathrm{L}$

Figure 1: Single span suspension bridge model.

\subsection{Two-field formulation}

Assuming zero horizontal displacement for the bridge deck, as well as zero transversal displacement and negligible longitudinal displacement for the cables, the motion of the bridge is described by means of two displacement functions (Fig. 2): vertical deflection $w_{d}(x, t)$ and twist rotation $\vartheta_{d}(x, t)$ around the longitudinal centerline of the deck, $t$ denoting time. The equations of motion are derived by means of Hamilton's principle $\delta \int_{t_{1}}^{t_{2}}(T-V+W) d t=0$, where, $T$ and $V$ are the total kinetic and potential energies of the system, respectively, $W$ is the work done by external forces and $t_{1}$ and $t_{2}$ are arbitrary time instants.

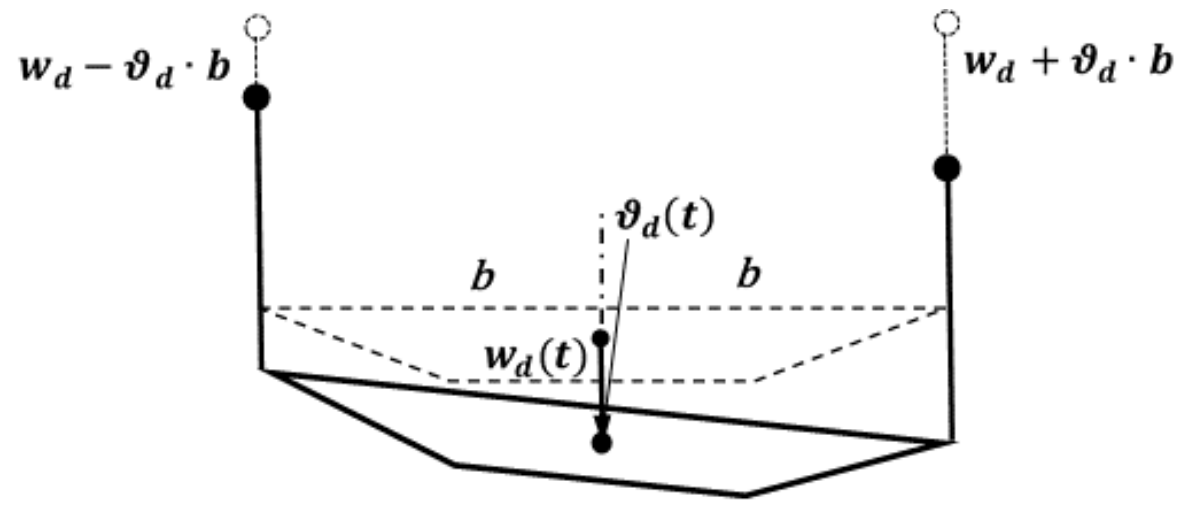

Figure 2: Kinematics of the cross section.

The equations of motion of the suspension bridge with the associated boundary conditions are readily derived. In particular, the following dimensionless equations are written by splitting on different rows the linear, quadratic and cubic components of the operators. 


$$
\begin{aligned}
& \text { flexural : }\left\{\begin{array}{c}
\frac{d^{2} \widetilde{w}_{d}}{d \tau^{2}}+\mu^{2} \cdot \widetilde{w}_{d}{ }^{\prime}-\widetilde{w}_{d}^{\prime \prime}+\lambda_{1}{ }^{2} \tilde{h}_{w}+ \\
-\lambda_{2}{ }^{2} \cdot\left[\widetilde{h}_{w} \cdot \widetilde{w}_{d}^{\prime \prime}+\tilde{h}_{\vartheta} \cdot \tilde{\vartheta}_{d}^{\prime \prime}-\frac{1}{2}\left(\tilde{h}_{w^{\prime} w^{\prime}}+\tilde{h}_{\vartheta^{\prime} \vartheta^{\prime}}\right)\right]+ \\
-\lambda_{3}{ }^{2} \cdot\left[\frac{1}{2}\left(\tilde{h}_{w^{\prime} w^{\prime}}+\tilde{h}_{\vartheta^{\prime} \vartheta^{\prime}}\right) \cdot \widetilde{w}_{d}^{\prime \prime}+\tilde{h}_{w^{\prime} \vartheta^{\prime}} \cdot \tilde{\vartheta}_{d}{ }^{\prime \prime}\right]
\end{array}\right\}=\tilde{q}(\xi, \tau) \\
& \text { torsional }:\left\{\begin{array}{c}
\widetilde{J}_{t} \cdot \frac{d^{2} \widetilde{\vartheta}_{d}}{d t^{2}}+\frac{\beta^{2}}{\chi^{2}} \cdot \tilde{\vartheta}_{d}{ }^{\prime v}-\left(1+\beta^{2}\right) \cdot \tilde{\vartheta}_{d}{ }^{\prime \prime}+\lambda_{1}{ }^{2} \widetilde{h}_{\vartheta}+ \\
-\lambda_{2}{ }^{2} \cdot\left[\tilde{h}_{\vartheta} \cdot \widetilde{w}_{d}{ }^{\prime \prime}+\tilde{h}_{w} \cdot \tilde{\vartheta}_{d}{ }^{\prime \prime}-\tilde{h}_{w^{\prime} \vartheta^{\prime}}\right]+ \\
-\lambda_{3}{ }^{2} \cdot\left[\tilde{h}_{w^{\prime} \vartheta^{\prime}} \cdot \widetilde{w}_{d}{ }^{\prime \prime}+\frac{1}{2}\left(\tilde{h}_{w^{\prime} w^{\prime}}+\tilde{h}_{\vartheta^{\prime} \vartheta^{\prime}}\right) \cdot \tilde{\vartheta}_{d}{ }^{\prime \prime}\right]
\end{array}\right\}=\widetilde{m}(\xi, \tau)
\end{aligned}
$$

A set of non-dimensional variables and parameters have been introduced having expressions

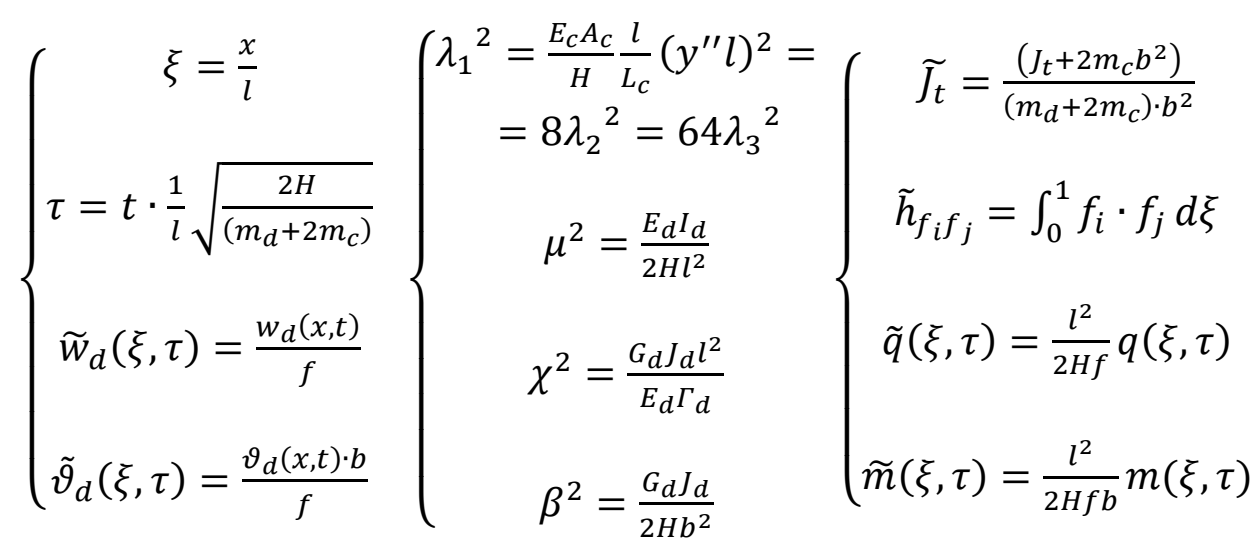

where: $x$ is the bridge axis coordinate; $l$ the main span length; $t$ the time variable; $H$ the initial horizontal component of cables' tension (due to dead loads only); $m_{d}$ and $m_{c}$ the deck and single cable mass per unit length, respectively; $w_{d}(x, t)$ and $\vartheta_{d}(x, t)$ the flexural and torsional motion of the of the deck axis, respectively; $f$ the initial cables sag; $b$ the half width of the deck section.

The Irvine parameter $\lambda_{1}{ }^{2}$ of the main cables [6], which has a crucial role in determining the eigen-properties of the bridge, depends also upon the axial stiffness of the cables system $E_{c} A_{c}$, the cables initial length $L_{c}$ and the initial cables curvature, assumed of parabolic shape $y^{\prime \prime}=$ $8 f / l^{2}$. Notice that this latter assumption allows the simple form of the quadratic $\lambda_{2}{ }^{2}$ and cubic $\lambda_{3}{ }^{2}$ Irvine terms. Other relevant non-dimensional parameters are $\mu^{2}$ (Steinman's stiffness factor [2]) and $\beta^{2}$, that reflect the relative flexural $\left(E_{d} I_{d}\right)$ and primary (St. Venant) torsional $\left(G_{d} J_{d}\right)$ stiffnesses of the girder, respectively. In order to analyze also the cases of bridge decks endowed with small primary torsional stiffness, the so-called warping coefficient is introduced, reflecting the ratio between the primary $\left(G_{d} J_{d}\right)$ and secondary (Vlasov-Wagner) torsional stiffness $\left(E_{d} \Gamma_{d}\right)$. We can define a dimensionless equivalent torsional inertia per unit length dependent both on the cables and deck contribution $J_{t}$. The so called non-local stiffening operators $\tilde{h}_{f_{i} f_{j}}\left(\right.$ e.g. $\left.\tilde{h}_{w^{\prime} \vartheta},=\int_{0}^{1} \widetilde{w}_{d}^{\prime}(\xi, \tau) \cdot \tilde{\vartheta}_{d}^{\prime}(\xi, \tau) d \xi\right)$ represent stiffness contributions coming from the 
main cables tension increment (stiffening behavior). Finally, we need to introduce some dimensionless external forces normalizing the generalized vertical force $q(\xi, \tau)$ and torsional couple $m(\xi, \tau)$ acting on the bridge deck axis.

\subsection{A brief note on slackening}

Slackening of hangers could have an important effect on the structural response mainly during large oscillations, because it generates a strong variation of the actual stiffness of the structural system. The introduction of an accurate constitutive model able to capture the linear elastic response of hangers in tension and their slackening in compression generally requires to increase the number of the fields from 2 to 4 [32-34]. Hence, when referring to the simplest 2field perfectly tenso-rigid hangers model, a limit condition able to distinguish between taut and slack hangers must be introduced. To this purpose, we consider a parameter that defines the dimensionless cables mass as function of the deck one.

$$
\widetilde{m}_{d}=\frac{m_{d}}{\left(m_{d}+2 m_{c}\right)}=1-\frac{2 m_{c}}{\left(m_{d}+2 m_{c}\right)}=1-2 \tilde{m}_{c}
$$

The critical conditions for the initiation of slackening correspond to that amplitude that induces null actions in the cables system, that is.

$$
\begin{gathered}
\tilde{F}_{d}(\xi, \tau)=8 \cdot \widetilde{m}_{d}+\left(1-\widetilde{m}_{d}\right) \cdot \frac{d^{2} \widetilde{w}_{d}}{d \tau^{2}}(\xi, \tau)-\widetilde{w}_{d}{ }^{\prime \prime}(\xi, \tau)+\lambda_{1}{ }^{2} \tilde{h}_{w}=0 \\
\tilde{C}_{d}(\xi, \tau)=\left(1-\widetilde{m}_{d}\right) \cdot \frac{d^{2} \widetilde{\vartheta}_{d}}{d \tau^{2}}(\xi, \tau)-\tilde{\vartheta}_{d}^{\prime \prime}(\xi, \tau)+\lambda_{1}{ }^{2} \tilde{h}_{\vartheta}=0
\end{gathered}
$$

Let's now exploit the modal expansion of the actual structural response.

$$
\begin{gathered}
\widetilde{w}_{d}(\xi, \tau)=\sum_{n=1}^{\infty} W_{n}(\xi) z_{n}(\tau)=\sum_{n=1}^{\infty} W_{n}(\xi) \cdot\left\{Z_{n} \cdot \exp \left(i \cdot \tilde{\Omega}_{w, n} \cdot \tau\right)+c . c .\right\} \\
\tilde{\vartheta}_{d}(\xi, \tau)=\sum_{m=1}^{\infty} \Theta_{m}(\xi) \gamma_{m}(\tau)=\sum_{m=1}^{\infty} \Theta_{m}(\xi) \cdot\left\{\Gamma_{m} \cdot \exp \left(i \cdot \tilde{\Omega}_{\vartheta, m} \cdot \tau\right)+c . c .\right\}
\end{gathered}
$$

Being the general dimensionless circular frequency defined as:

$$
\tilde{\Omega}=\Omega \cdot l \sqrt{\frac{\left(m_{d}+2 m_{c}\right)}{2 H}}
$$

Hence the critical amplitude and curvature can be found.

$$
\begin{gathered}
\tilde{F}_{d, n}=0 \Leftrightarrow Z_{n, c r}=8 \cdot \widetilde{m}_{d} \cdot \min \left\{\left|\left(1-\widetilde{m}_{d}\right) \widetilde{\Omega}_{w, n}{ }^{2} \cdot W_{n}(\xi)+W_{n}{ }^{\prime \prime}(\xi)-\lambda_{1}{ }^{2} \tilde{h}_{W, n}\right|^{-1}\right\} \\
\tilde{C}_{d, m}=0 \Leftrightarrow \Theta_{m, c r}{ }^{\prime \prime}(\xi)=\lambda_{1}{ }^{2} \widetilde{h}_{\Theta, m}-\left(1-\widetilde{m}_{d}\right) \widetilde{\Omega}_{\vartheta, m}{ }^{2} \cdot \Theta_{m}(\xi)
\end{gathered}
$$

In this way a critical amplitude threshold can be defined just for the flexural vibration component, with the same expression found by Luco and Turmo [5]. 


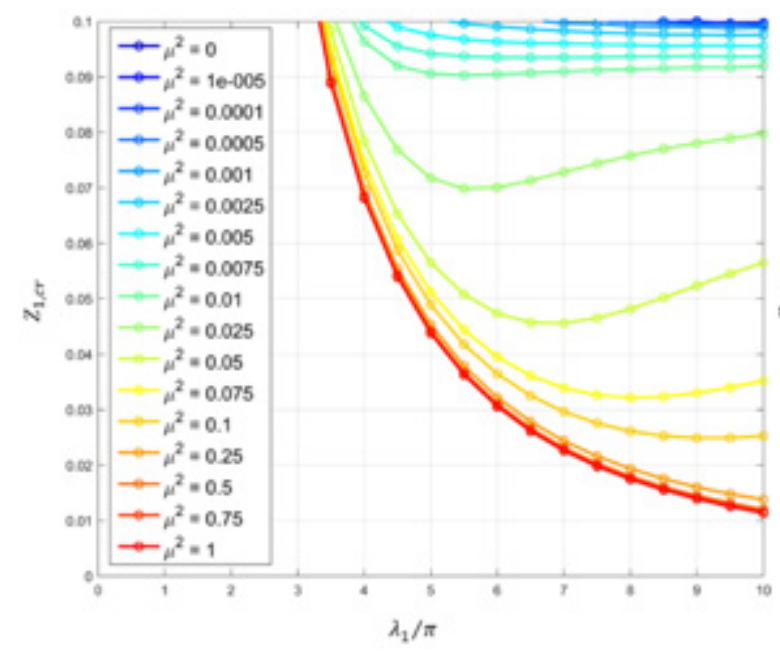

Figure 3: Dimensionless antinode displacement at first slackening for flexural mode 1 , with $\widetilde{m}_{d}=0.85$.

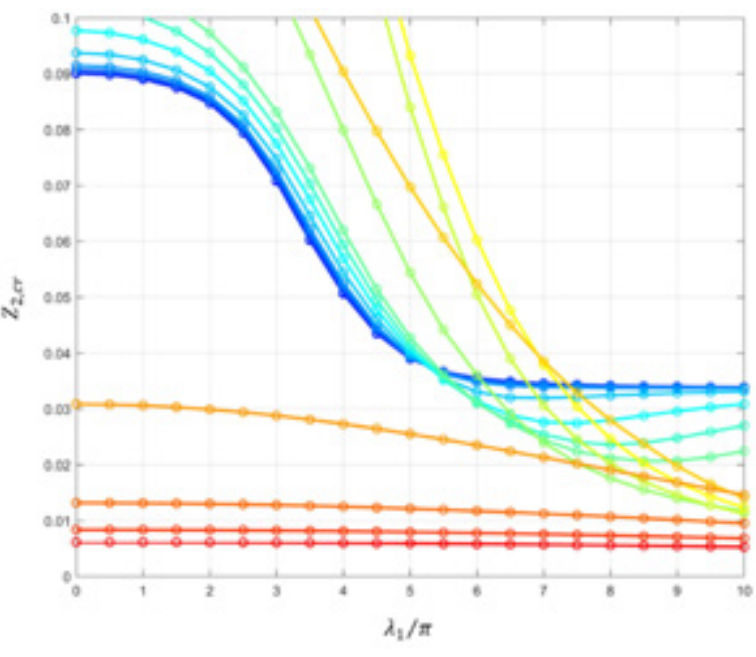

Figure 4: Dimensionless antinode displacement at first slackening for flexural mode 2 , with $\widetilde{m}_{d}=0.85$.

As it can be seen from (Fig. 3), a decrease into the deck flexural stiffness requires higher antinode displacements for slackening onset. Conversely, by increasing the cables inextensibility we get decreasing limits only if deck stiffness is high enough. In fact, for limited values of $\mu^{2}$ the dependence upon $\lambda_{1}^{2}$ does not decrease monotonically.

Slackening threshold amplitudes rapidly decreases as the modal order increases (Fig. 4), since the up-lifting regions extend to a larger part of the deck.

\section{THE LINEAR EIGENVALUE PROBLEMS}

In this paper the solution of the equations of motion is sought through modal expansions previously introduced in (7) and (8). This requires the knowledge of the eigensolutions.

By enforcing the suitable boundary conditions for the flexural hinges $\left\{\widetilde{w}_{d}(0, \tau)=\widetilde{w}_{d}(1, \tau)=\right.$ $\left.0 ; \widetilde{w}_{d}^{\prime \prime}(0, \tau)=\widetilde{w}_{d}^{\prime \prime}(1, \tau)=0\right\}$ and for the torsional forks $\left\{\tilde{\vartheta}_{d}(0, \tau)=\tilde{\vartheta}_{d}(1, \tau)=0\right.$; $\left.\tilde{\vartheta}_{d}^{\prime \prime}(0, \tau)=\tilde{\vartheta}_{d}^{\prime \prime}(1, \tau)=0\right\}$, one gets the natural frequencies and the symmetric and antisymmetric modes. The expressions obtained are identical to those reported by Cevik and Pakdemirli [22]. It is worth noting that the symmetric modes include trigonometric and hyperbolic functions, conversely the skew-symmetric ones are represented by simple sinusoidal shapes.

The influence of the main parameters $\lambda_{1}{ }^{2}, \chi^{2}, \mu^{2}$, and $\beta^{2}$ has been investigated through a parametric analysis, based on the available data collected from the literature [5,12,35-44]. Besides realistic values, also extreme conditions are considered such as $\lambda_{1}{ }^{2}=0$ (flat cables), $\lambda_{1}{ }^{2}=\infty$ (inextensible cables), $\mu^{2}=0$ (flexible deck), $\chi^{2}=0$ (rigid warping), $\chi^{2}=\infty$ (free warping), $\beta^{2}=0$ (flexible deck).

For the sake of brevity, we will report just the results obtained for the second symmetric mode of vibrations, that shows more interesting features than the first one. Figure 5 shows the flexural eigen-frequency, as a function of the dimensionless deck bending stiffness, for different values of the Irvine parameter. Figure 6 is referred to torsional eigen-frequency, in the case of free warping. 


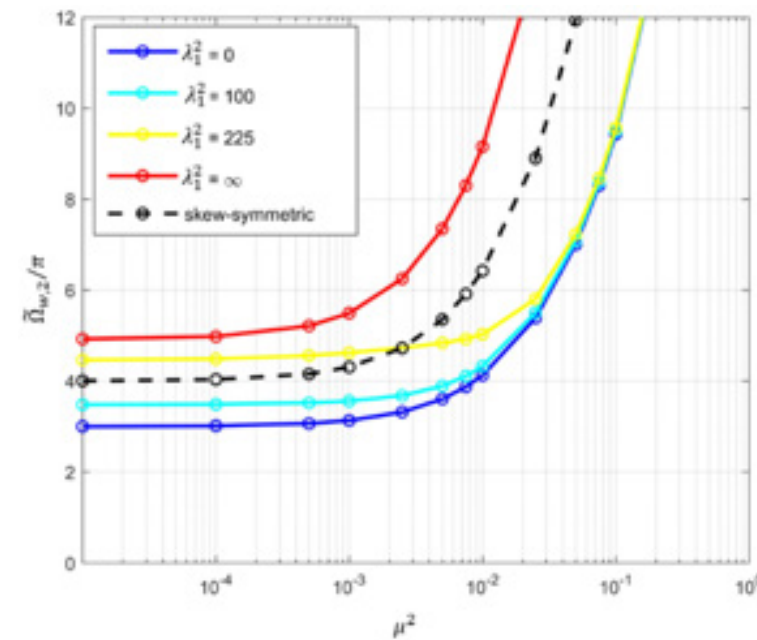

Figure 5: Circular eigen-frequencies of flexural symmetric mode 2 .

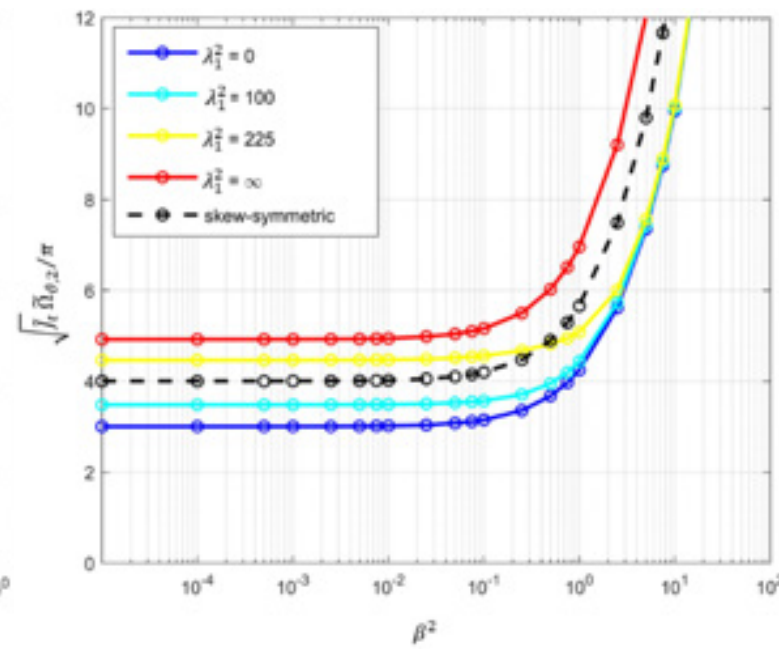

Figure 6: Circular eigen-frequencies of torsional symmetric mode 2 for $\chi^{2}=\infty$.

Notice that the upward (negative) displacement increases as the Irvine parameter $\lambda_{1}{ }^{2}$ does, since as cables become more and more inextensible, their initial parabolic shape turns out to be much more influent on deck deformation. Further as $\lambda_{1}{ }^{2}=\infty$, the modal shapes coalesce approximately to a single sinusoidal curve for any value of $\mu^{2}$ and $\beta^{2}$. Similarly, if higher modes are considered $(n>4)$, one gets modal shapes closer to sinusoidal ones since the hyperbolic cosine contribution becomes negligible in the mode shape, meaning that deck stiffness is less influent on bridge vibration. Concerning torsional modes only, passing from $\chi^{2}=\infty$ to $\chi^{2}=$ 0 we noticed that the deck relative stiffness becomes much more relevant in the latter case.

Furthermore, for each value of the parameter $\mu^{2}$ and $\beta^{2}$ there exists a critical value for $\lambda_{1}{ }^{2}$ beyond which a modal inversion occurs, that is, the symmetric $\mathrm{n}^{\text {th }}$-mode appears 'earlier' than the skew-symmetric one. Indeed, in Figs. 5-6, circular eigen-frequencies of skew-symmetric mode are lower than the ones of symmetric mode just for some combination of structural parameters $\mu^{2}, \beta^{2}$ with $\lambda_{1}^{2}$.

From the mode shapes represented in (Fig. 7-8) it is evident that increasing the relative deckto-cable stiffness (flexural and torsional) the positive antinodal points move away from the midspan. 


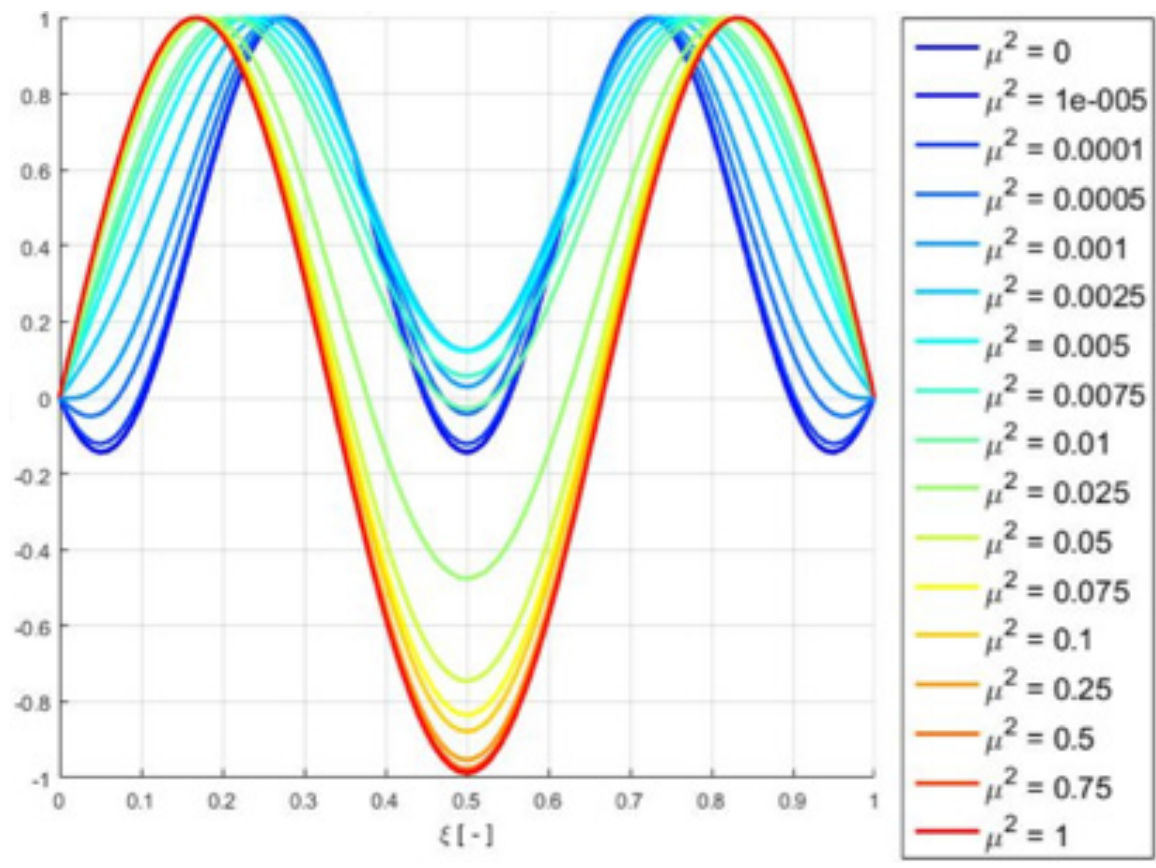

Figure 7: Modal shape of flexural symmetric mode 2 for $\lambda_{1}{ }^{2}=225$.

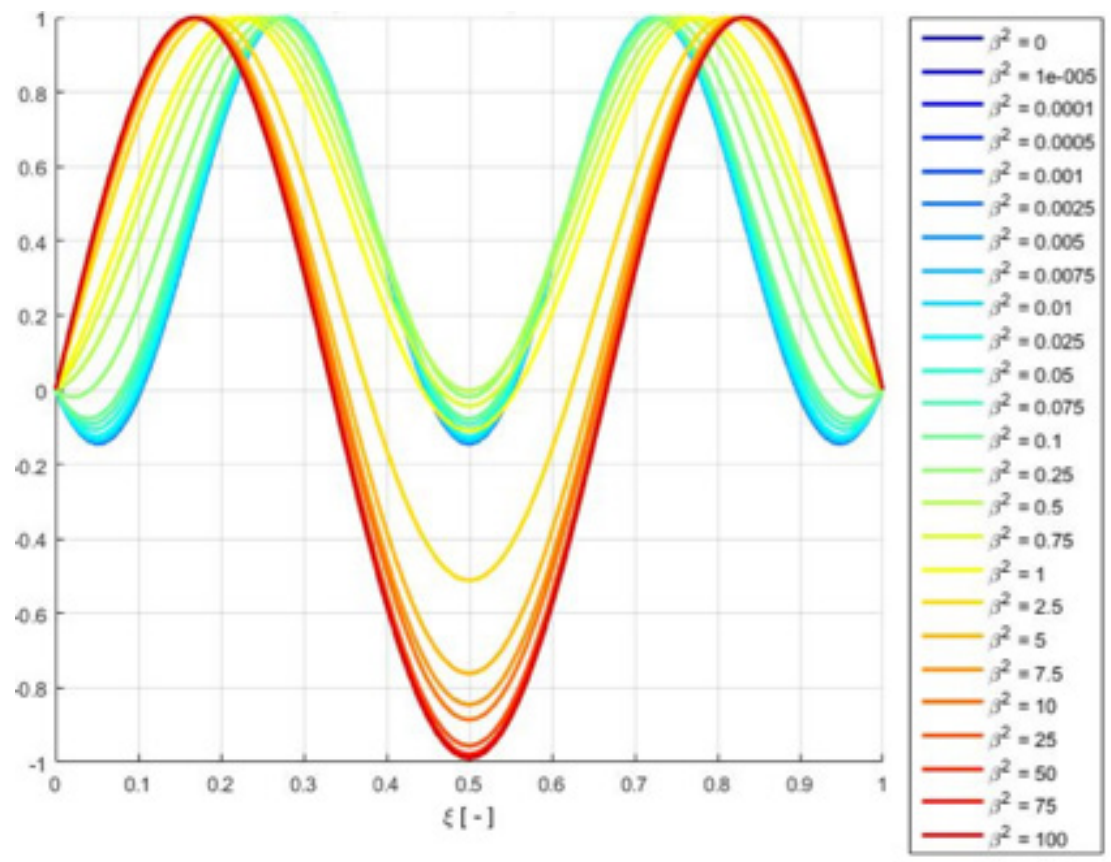

Figure 8: Modal shape of torsional symmetric mode 2 for $\lambda_{1}{ }^{2}=225$ and for $\chi^{2}=\infty$. 


\section{THE AEROELASTIC STRUCTURAL MODEL}

\subsection{Modal projection of equations of motion}

A Galerkin projection of the two equations of motion is obtained on the basis of modal analysis. Multiplying the flexural and torsional equation of motion by the respective modal shape of interest and integrating over the whole span of the deck we get

$$
\begin{gathered}
\left\{\begin{array}{c}
M_{w, n} \cdot \ddot{z}_{n}+D_{w, n} \cdot \dot{z}_{n}+D_{w \vartheta, n m} \cdot \dot{\gamma}_{m}+K_{w, n}^{(L)} \cdot z_{n}+K_{w \vartheta, n m}^{(L)} \cdot \gamma_{m}+ \\
+K_{w, n}^{(Q)} \cdot z_{n}{ }^{2}+K_{w \vartheta, n m}^{(Q)} \cdot \gamma_{m}{ }^{2}+ \\
+K_{w, n}^{(C)} \cdot z_{n}{ }^{3}+K_{w \vartheta, n m}^{(C)} \cdot z_{n} \cdot \gamma_{m}{ }^{2}
\end{array}\right\}=\Gamma_{w, n} \\
\left\{\begin{array}{c}
\widetilde{J}_{t} M_{\vartheta, m} \cdot \ddot{\gamma}_{m}+D_{\vartheta, m} \cdot \dot{\gamma}_{m}+D_{\vartheta w, m n} \cdot \dot{z}_{n}+K_{\vartheta, m}^{(L)} \cdot \gamma_{m}+K_{\vartheta w, m n}^{(L)} \cdot z_{n}+ \\
+K_{\vartheta w, m n}^{(Q)} \cdot \gamma_{m} \cdot z_{n}+ \\
+K_{\vartheta, m}^{(C)} \cdot \gamma_{m}{ }^{3}+K_{\vartheta w, m n}^{(C)} \cdot \gamma_{m} \cdot z_{n}{ }^{2}
\end{array}\right\}=\Gamma_{\vartheta, m}(\xi, \tau)(13
\end{gathered}
$$

Linear terms, that represent the aeroelastic contributions coming from wind-structure interaction, are added to the right hand side of (12-13), as better explained in the following section. Note that the torsional modal equation of motion is characterized by the presence of a second order coupling term. This is due to the fact that rotations of the deck introduce an asymmetric response of the two main cables, but this response depends strongly on the flexural amplitude of vibration that affect the stiffness of the cables system. This property of the system governs stability features of the dynamic response.

\subsection{Introduction to the classical formulation for Flutter}

For the sake of simplicity, the wind-structure interaction has been modeled by means of the socalled Flutter Derivatives approach first proposed by Simiu and Scanlan [14,15] in order to adapt Theodorsen's Theory [16] to bridge deck sections. Now, without any loss of generality, the deck's section will be modelled as an airfoil.

Let's write the aerodynamic lift and couple acting on the axis of the bridge's deck.

$$
\begin{gathered}
L(x, t)=\frac{1}{2} \rho_{a} U^{2} 2 b\left\{K_{w} H_{1}^{*} \frac{1}{U} \cdot \dot{w}_{d}+K_{\vartheta} H_{2}^{*} \frac{2 b}{U} \cdot \dot{\theta}_{d}+K_{\vartheta}^{2} H_{3}^{*} \cdot \theta_{d}+K_{w}^{2} H_{4}^{*} \frac{1}{2 b} \cdot w_{d}\right\} \\
M(x, t)=\frac{1}{2} \rho_{a} U^{2}(2 b)^{2}\left\{K_{w} A_{1}^{*} \frac{1}{U} \cdot \dot{w}_{d}+K_{\vartheta} A_{2}^{*} \frac{2 b}{U} \cdot \dot{\theta}_{d}+K_{\vartheta}^{2} A_{3}^{*} \cdot \theta_{d}+K_{w}^{2} A_{4}^{*} \frac{1}{2 b} \cdot w_{d}\right\}
\end{gathered}
$$

Where $\rho_{a}$ is the air density, $U$ the mean wind speed, $H_{(\cdot)}^{*}$ and $A_{(\cdot)}^{*}$ the mentioned Flutter Derivatives. The latter ones have been defined analytically for the airfoil by Theodorsen [16] as functions of $C_{w}, C_{\theta}$ respectively flexural and torsional Theodorsen's complex functions depending upon the actual reduced frequency $K_{(\cdot)}$.

$$
C_{(\cdot)}=C\left(K_{(\cdot)}\right)=F\left(K_{(\cdot)}\right)+i \cdot G\left(K_{(\cdot)}\right)
$$




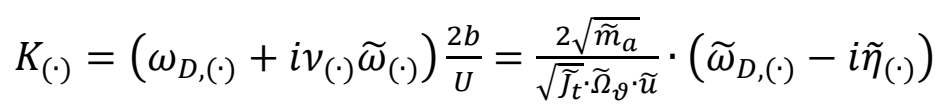

Where the term $+i v_{(\cdot)} \widetilde{\omega}_{(\cdot)}$ comes from the generalization of the time varying terms in the modal projection $(7,8)$, e. g. $z_{n}(\tau)=Z_{n} \cdot \exp \left\{\left(-v_{w, n} \widetilde{\omega}_{w, n}+\mathrm{i} \widetilde{\omega}_{\mathrm{D}, \mathrm{w}, \mathrm{n}}\right) \tau\right\}+$ c. c., taking into account damping. Being $\widetilde{\omega}_{D,(\cdot)}=\widetilde{\omega}_{(\cdot)} \sqrt{1-v_{(\cdot)}^{2}}$ the aeroelastic damped modal circular frequency, function of $\widetilde{\omega}_{(\cdot)}$ the aeroelastic modal circular frequency and $v_{(\cdot)}$ the aeroelastic modal viscous damping ratio, both variable with the wind speed level.

For Theodorsen's airfoil the following expression for the Flutter Derivatives are valid $[15,16]$.

$$
\left\{\begin{array} { c } 
{ H _ { 1 } ^ { * } = - 2 \pi \frac { F _ { w , n } } { K _ { w , n } } } \\
{ H _ { 2 } ^ { * } = - \frac { \pi } { 2 } \frac { 1 } { K _ { \vartheta , m } } ( 1 + 4 \frac { G _ { \vartheta , m } } { K _ { \vartheta , m } } + F _ { \vartheta , m } ) } \\
{ H _ { 3 } ^ { * } = - \pi \frac { 1 } { K _ { \vartheta , m } ^ { 2 } } ( 2 F _ { \vartheta , m } - \frac { 1 } { 2 } G _ { \vartheta , m } K _ { \vartheta , m } ) } \\
{ H _ { 4 } ^ { * } = \frac { \pi } { 2 } ( 1 + 4 \frac { G _ { w , n } } { K _ { w , n } } ) }
\end{array} \quad \left\{\begin{array}{c}
A_{1}^{*}=\frac{\pi}{2} \frac{F_{w, n}}{K_{w, n}} \\
A_{2}^{*}=-\frac{\pi}{2} \frac{1}{K_{\vartheta, m}^{2}}\left(\frac{1}{4} K_{\vartheta, m}-G_{\vartheta, m}-\frac{1}{4} K_{\vartheta, m} F_{\vartheta, m}\right) \\
A_{3}^{*}=\frac{\pi}{2} \frac{1}{K_{\vartheta, m}^{2}}\left(\frac{1}{32} K_{\vartheta, m}^{2}+F_{\vartheta, m}-\frac{1}{4} K_{\vartheta, m} G_{\vartheta, m}\right) \\
A_{4}^{*}=-\frac{\pi}{2} \frac{G_{w, n}}{K_{w, n}}
\end{array}\right.\right.
$$

For a generic deck's cross section, Flutter Derivatives are defined either experimentally (through Wind Tunnel Tests) or numerically (by means of CFD analyses [45]). In both cases, only discretized curves are available.

In the adopted model, aeroelastic forces introduces additional linear terms only. For the sake of completeness, it is worth noting that some examples of non-linear models for the aerodynamics loads can be retrieved in the literature [46]; moreover, further examples of non-linear aeroelastic loads can be found in the context of quasi-steady formulations [47]. In the present case, the linear equations of motion also become coupled and the system loses its symmetries in stiffness and damping, thus becoming susceptible of static divergence and flutter dynamic instability. The dimensionless modal linear coefficients due to aeroelastic effects can be written as

$$
\left\{\begin{array}{c}
D_{w, n}=\left(2 \Delta_{w, n} \cdot \tilde{\Omega}_{w, n}-\frac{1}{\pi} \sqrt{\widetilde{J}_{t} \cdot \widetilde{m}_{a}} \cdot \tilde{\Omega}_{\vartheta, m} \cdot \tilde{u} \cdot K_{w, n} \cdot H_{1}^{*}\right) \cdot M_{w, n} \\
D_{w \vartheta, n m}=-\frac{2}{\pi} \sqrt{\widetilde{J_{t}} \cdot \widetilde{m}_{a}} \cdot \tilde{\Omega}_{\vartheta, m} \cdot \tilde{u} \cdot K_{\theta, m} \cdot H_{2}^{*} \cdot \tilde{h}_{W_{n}, \Theta_{m}} \\
K_{w, n}^{(L)}=\left(\tilde{\Omega}_{w, n}^{2}-\frac{1}{2 \pi}\left(\sqrt{\widetilde{J}_{t}} \cdot \widetilde{\Omega}_{\vartheta, m} \cdot \tilde{u} \cdot K_{w, n}\right)^{2} \cdot H_{4}^{*}\right) \cdot M_{w, n} \\
K_{w \vartheta, n m}^{(L)}=-\frac{1}{\pi}\left(\sqrt{\widetilde{J}_{t}} \cdot \widetilde{\Omega}_{\vartheta, m} \cdot \tilde{u} \cdot K_{\vartheta, m}\right)^{2} \cdot H_{3}^{*} \cdot \tilde{h}_{W_{n}, \Theta_{m}}
\end{array}\right.
$$




$$
\left\{\begin{array}{c}
D_{\vartheta, m}=\left(2 \Delta_{\vartheta, m} \cdot \widetilde{J_{t}} \cdot \tilde{\Omega}_{\vartheta, m}-\frac{4}{\pi} \sqrt{\widetilde{J_{t}} \cdot \widetilde{m}_{a}} \cdot \tilde{\Omega}_{\vartheta, m} \cdot \tilde{u} \cdot K_{\vartheta, m} \cdot A_{2}^{*}\right) \cdot M_{\vartheta, m} \\
D_{\vartheta w, m n}=-\frac{2}{\pi} \sqrt{\widetilde{J_{t}} \cdot \widetilde{m}_{a}} \cdot \tilde{\Omega}_{\vartheta, m} \cdot \tilde{u} \cdot K_{w, n} \cdot A_{1}^{*} \cdot \tilde{h}_{W_{n}, \theta_{m}} \\
K_{\vartheta, m}^{(L)}=\left(\widetilde{J_{t}} \cdot \tilde{\Omega}_{\vartheta, m}^{2}-\frac{2}{\pi}\left(\sqrt{\widetilde{J}_{t}} \cdot \tilde{\Omega}_{\vartheta, m} \cdot \tilde{u} \cdot K_{\vartheta, m}\right)^{2} \cdot A_{3}^{*}\right) \cdot M_{\vartheta, m} \\
K_{\vartheta w, m n}^{(L)}=-\frac{1}{\pi}\left(\sqrt{\widetilde{J}_{t}} \cdot \tilde{\Omega}_{\vartheta, m} \cdot \tilde{u} \cdot K_{w, n}\right)^{2} \cdot A_{4}^{*} \cdot \tilde{h}_{W_{n}, \Theta_{m}}
\end{array}\right.
$$

The additional aerodynamic mass $\widetilde{m}_{a}$ and torsional inertia $\widetilde{J_{a}}$ give negligible contributions with respect to structural ones $[5,12,35-44]$ hence they don't appear in the definition of modal masses.

In view of its importance in the stability analysis, the analytical expression of the quadratic coupling term is also reported.

$$
K_{\vartheta w, m n}^{(Q)}=\lambda_{Q}^{2} \cdot\left(2 \cdot \tilde{h}_{\theta_{m}} \cdot \tilde{h}_{W_{n}^{\prime}, \theta_{m}^{\prime}}+\tilde{h}_{W_{n}} \cdot \tilde{h}_{\Theta_{m}^{\prime 2}}\right)
$$

The parameter $\Delta_{(\cdot)}$ is the structural modal viscous damping ratio, assumed for simplicity constant and equal to $0.5 \%$, whilst $\tilde{u}$ represents the mean wind speed $U$ normalized with respect to the static divergence one $U_{D(\vartheta, \mathrm{m})}$ in correspondence of the same structural parameters and torsional mode of interest.

$$
U_{D(\vartheta, m)}=\sqrt{\widetilde{J}_{t}} \widetilde{\Omega}_{\vartheta, m} \cdot \sqrt{\frac{2 H}{\pi \rho_{a} l^{2}}}
$$

Notice the difference between the groups $\left[\tilde{\Omega}_{(\cdot)} ; \Delta_{(\cdot)}\right]$ and $\left[\widetilde{\omega}_{(\cdot)} ; v_{(\cdot)}\right]$ which collect the circular frequency and the modal viscous damping ratio coming from the structural model (as in Section 3 ) and the complete aeroelastic one, respectively. Consequently, the variables of the first group will be constant and those of the second one will vary with the mean wind speed level.

\section{FORMULATION OF STABILITY ANALYSIS}

The main purpose of the analysis is to assess the stability of the dynamic response of the structural system. First, the classical Flutter analysis is exploited to define a critical wind speed beyond which the structural response would diverge in time. Next, the introduction of a perturbed system of equations will allow accounting for the parametric second order coupling of the flexural-torsional motion. Notice that the governing equations are linear in both the analyses.

\subsubsection{Flutter critical threshold}

Wind speed corresponding to the flutter onset is controlled by the following linear contribution to the equations governing the non-linear aeroelastic structural model formulated in Section 4.2.

$$
\begin{aligned}
& M_{w, n} \cdot \ddot{z}_{n}+D_{w, n} \cdot \dot{z}_{n}+D_{w \vartheta, n m} \cdot \dot{\gamma}_{m}+K_{w, n}^{(L)} \cdot z_{n}+K_{w \vartheta, n m}^{(L)} \cdot \gamma_{m}=0 \\
& \tilde{J}_{t} M_{\vartheta, m} \cdot \ddot{\gamma}_{m}+D_{\vartheta, m} \cdot \dot{\gamma}_{m}+D_{\vartheta w, m n} \cdot \dot{z}_{n}+K_{\vartheta, m}^{(L)} \cdot \gamma_{m}+K_{\vartheta w, m n}^{(L)} \cdot z_{n}=0
\end{aligned}
$$


The critical condition for Flutter onset can be the change of sign, from positive to negative, for the net torsional or flexural damping.

\subsubsection{Internal Parametric resonance}

A recent work by Arioli and Gazzola [24], as previous ones by different authors [21,22] investigates internal parametric resonance potentially suffered by suspension bridges. In order to catch this phenomenon, it is necessary to consider the fully non-linear equation of motion, hereby for the first time supplemented by the aeroelastic operator.

\subsubsection{Vortex-shedding modelling}

In many real systems the parameters into the governing equations may vary periodically in time [48]. This is not generally the case when dealing with suspension bridges, since structural parameters are fixed and can vary only in a very long period. Nevertheless, due to the peculiar non-linear coupling of the governing equations and accounting for aeroelastic phenomena, one can ascertain that parametric resonance may occur in the presence of a periodic external action proportional to some kinematic parameter of the systems, as those produced by the vortexshedding, represented by means of a sinusoidal transverse force $[14,15]$ :

$$
F_{L}=\frac{1}{2} \cdot \rho_{a} \cdot 2 b \cdot U^{2} \cdot C_{L} \cdot \sin \left(\omega_{V S} \cdot t\right)
$$

where $\omega_{V S}$ is the frequency of vortex shedding and $C_{L}$ is the lift coefficient, which basically depends on the cross section shape, on the Reynolds number and on the surface roughness. The equivalent modal lift force has the following dimensionless format.

$$
\begin{gathered}
\Gamma_{w, n}=\Gamma_{0} \cdot \tilde{h}_{W_{n}} \cdot \sin \left(\widetilde{\omega}_{V S} \cdot \tau\right) \\
\Gamma_{0}=2 \tilde{b} \cdot \tilde{c}_{L} \cdot{\widetilde{J_{t}}} \cdot \tilde{\Omega}_{\vartheta, m}{ }^{2} \cdot \tilde{u}^{2}
\end{gathered}
$$

where we have introduced the non-dimensional counterpart for the deck's width-to-sag ratio and the normalized lift coefficient with respect to the analytical one characterizing thin airfoils [14]:

$$
\begin{gathered}
\tilde{b}=b / f \\
\tilde{c}_{L}=C_{L}\left(\theta_{d}\right) / 2 \pi
\end{gathered}
$$

It is worth noting that the stability analyses will be carried out by considering the forcing frequency as an independent variable, while when examining the results the Strouhal relationship is considered $[14,15]$ :

$$
\omega_{V S}=2 \pi \cdot f_{V S}=2 \pi \cdot S t \cdot U / D
$$

where $D$ is the depth of the deck's cross section.

Moreover, the presence of a 'lock-in' region is accounted for by considering an interval of wind velocity, e.g. $\{0.8-1.3\} \cdot \tilde{u}_{\text {lock-in }}$, in which there is a synchronism between structural and vortex shedding frequency. The dimensionless format of Strouhal relationship reads:

$$
\begin{gathered}
\widetilde{\omega}_{V S}=\pi \cdot S t \cdot\left(\tilde{\alpha} / \sqrt{\widetilde{m}_{a}}\right) \cdot \sqrt{\widetilde{J}_{t}} \cdot \tilde{\Omega}_{\vartheta, m} \cdot \tilde{u} \\
\tilde{u}_{l o c k-i n}=\widetilde{\omega}_{w, n} /\left\{\pi \cdot S t \cdot\left(\tilde{\alpha} / \sqrt{\widetilde{m}_{a}}\right) \cdot \sqrt{\widetilde{J_{t}}} \cdot \widetilde{\Omega}_{\vartheta, m}\right\}
\end{gathered}
$$


where $\tilde{\alpha}=2 b / D$ is the aspect ratio of the cross section: from available bridges data $[5,12,35$ 44] $\tilde{\alpha}=3 \div 12$. Concerning the Strouhal number, we will consider $S t=0.1$ [49] associated to an airfoil.

Finally, we must remember that vortex-shedding is a self-limited phenomenon due to aerodynamic damping. Experimental investigations showed that oscillations of circular cylinders never overcome the $20 \%$ of the cross flow dimension of the body. Consequently an empirical upper bound for dimensionless vertical vibrations $\widetilde{\mathrm{w}}_{d}(\xi, \tau)$ should be taken about equal to $2 \%$ assuming $\tilde{\alpha}=6.4$ and $\tilde{b}=0.32$ from average values of [5,12,35-44].

\subsubsection{Perturbed system of equations}

In order to study the stability of the suspension bridge vibrations as long as dominant flexural motion occurs, the perturbation approach outlined in Hermann and Hauger [31] is adopted.

As first step, we assume a reference solution with small flexural vibrations and negligible torsional ones, so that we can neglect non-linear flexural contributions and assume a lift coefficient in correspondence of a null torsional angle.

$$
\begin{gathered}
z_{n}(\tau)<\varepsilon \Rightarrow z_{n}{ }^{2}(\tau) \cong 0 ; z_{n}{ }^{3}(\tau) \cong 0 \\
\gamma_{m}(\tau) \cong 0 \Rightarrow \tilde{c}_{L}=C_{L}\left(\theta_{d} \cong 0\right) / 2 \pi
\end{gathered}
$$

Hence, the linear equation of motion of a SDOF damped and periodically forced oscillator is found:

$$
M_{w, n} \cdot \ddot{z}_{n}+D_{w, n} \cdot \dot{z}_{n}+K_{w, n}^{(L)} \cdot z_{n}=\Gamma_{0} \cdot \tilde{h}_{W_{n}} \cdot \sin \left(\widetilde{\omega}_{V S} \cdot \tau\right)
$$

whose solution is given by the superposition of a homogenous-transient and a particular-steady integral. Since we are looking for unstable solutions, the transient conditions are not of interest and the steady state solution is defined through the complex dynamic amplification factor:

$$
\begin{aligned}
& z_{n, S S}(\tau)=z_{n, 0} \cdot \sin \left(\widetilde{\omega}_{V S} \cdot \tau-\varphi\right) \\
& z_{n, 0}=\Gamma_{0} \cdot \tilde{h}_{W_{n}} \cdot\left|H\left(\widetilde{\omega}_{V S}\right)\right|
\end{aligned}
$$

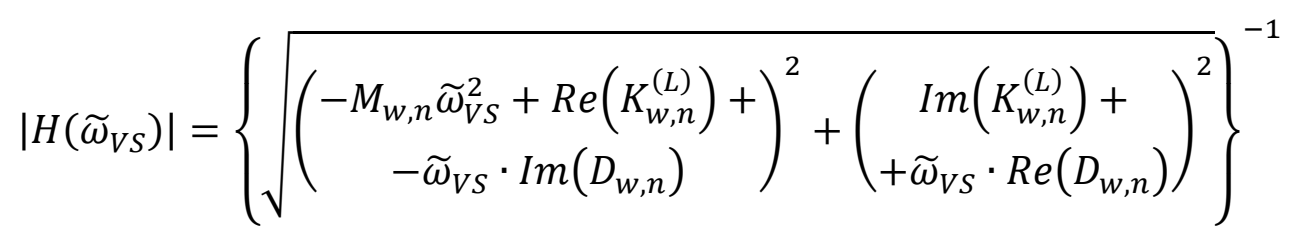

$$
\begin{aligned}
& \operatorname{tg}(\varphi)=\left(\begin{array}{c}
\operatorname{Im}\left(K_{w, n}^{(L)}\right)+ \\
+\widetilde{\omega}_{V S} \cdot \operatorname{Re}\left(D_{w, n}\right)
\end{array}\right) /\left(\begin{array}{c}
-M_{w, n} \widetilde{\omega}_{V S}^{2}+\operatorname{Re}\left(K_{w, n}^{(L)}\right)+ \\
-\widetilde{\omega}_{V S} \cdot \operatorname{Im}\left(D_{w, n}\right)
\end{array}\right)
\end{aligned}
$$

The second step of the analysis requires introducing a small perturbation to the actual steady response of the forced system, for both flexural and torsional DOF:

$$
\begin{gathered}
z_{n}(\tau)=z_{n, S S}(\tau)+z_{P, n}(\tau) \text { with } z_{P, n}(t)<\varepsilon \\
\gamma_{m}(\tau)=\gamma_{P, m}(\tau) \text { with } \gamma_{P, m}(\tau)<\varepsilon
\end{gathered}
$$

Then, by substituting the above definitions in the complete modal aeroelastic system of equations and by suitable linearization, one gets the so-called perturbed system.

$$
M_{w, n} \ddot{z}_{P, n}+D_{w, n} \dot{z}_{P, n}+D_{w \vartheta, n m} \dot{\gamma}_{P, m}+K_{w, n}^{(L)} z_{P, n}+K_{w \vartheta, n m}^{(L)} \gamma_{P, m}=0
$$


$\tilde{J}_{t} M_{\vartheta, m} \ddot{\gamma}_{P, m}+D_{\vartheta, m} \dot{\gamma}_{P, m}+D_{\vartheta w, m n} \dot{Z}_{P, n}+K_{\vartheta w, m n}^{(L)} z_{P, n}+\left\{K_{\vartheta, m}^{(L)}+K_{\vartheta w, m n}^{(Q)} z_{0} \sin \left(\widetilde{\omega}_{V S} \tau-\varphi\right)\right\} \gamma_{P, m}=0$

As previously mentioned, the second order term of torsional motion is dependent simultaneously and linearly on both motions, while the flexural one depends quadratically on the two independent contributions. This makes possible that a small but not vanishing vertical perturbation influences the torsional response due to a periodically varying structural parameter, even in a linearized formulation. The fact that it depends on the actual vortex-shedding excitation is hidden inside the terms $z_{n, 0}$ and $\varphi$, that are implicitly dependent on the lift coefficient since they are obtained as the solution of eq. (34). This kind of phenomenon can be classified as a parametric excitation problem. We have already stressed that, being a self-limiting phenomenon, vortex shedding can induce just small flexural perturbations. However, because their effects on torsional vibrations is amplified by the quadratic coupled modal stiffness $K_{\vartheta w, m n}^{(Q)}$, it will be of basic importance to understand its influence on the system response.

\section{STABILITY ANALYSIS}

We analyze the equations derived in the previous chapters in view of a stability assessment. The vortex-shedding phenomenon introduces a dynamic flexural perturbation, acting as a periodic variation on the torsional stiffness of the perturbed system.

\subsection{Mathieu equation for the homogeneous perturbed system}

Let's now consider the perturbed equations without accounting for the aeroelastic effects coming from wind-structure interaction, leading to an uncoupled system.

$$
\begin{gathered}
M_{w, n} \ddot{z}_{P, n}+D_{w, n} \dot{z}_{P, n}+K_{w, n}^{(L)} z_{P, n}=0 \\
\tilde{J}_{t} M_{\vartheta, m} \ddot{\gamma}_{P, m}+D_{\vartheta, m} \dot{\gamma}_{P, m}+\left\{K_{\vartheta, m}^{(L)}+K_{\vartheta w, m n}^{(Q)} z_{n, 0} \sin \left(\widetilde{\omega}_{V S} \tau-\varphi\right)\right\} \gamma_{P, m}=0
\end{gathered}
$$

Notice that the damping terms contain just the structural contribution that is positive and generally very small. Hence, we can say $z_{P, n}$ will not diverge in time.

A proper choice of the reference initial time instant allows us to shift the flexural perturbation acting on the torsional motion and substitute the sinus with a cosine function.

Next, we want to reduce the damped torsional equation to the so-called Mathieu format. In this way, we will be able to focus our attention on the Internal Parametric Resonance phenomenon, governed completely by the structural property of the system. After some rearrangements, we get the following equivalent damped Mathieu equation:

$$
\frac{d^{2}}{d \bar{\tau}^{2}} \gamma_{P, m}+\sigma \frac{d}{d \bar{\tau}} \gamma_{P, m}+\{\delta-2 \epsilon \cos (2 \bar{\tau})\} \gamma_{P, m}=0
$$

Where we have defined the following quantities:

$$
\begin{array}{rlrl}
\bar{\tau}=\left(\widetilde{\omega}_{V S} / 2\right) \cdot \tau & ; & \sigma=2 D_{\vartheta, m} /\left(\tilde{U}_{t} M_{\vartheta, m} \widetilde{\omega}_{V S}\right) \\
\delta=4\left(\widetilde{\Omega}_{\vartheta, m} / \widetilde{\omega}_{V S}\right)^{2} ; & \epsilon=2 \cdot\left(K_{\theta w, m n}^{Q} z_{n, 0}\right) /\left(\tilde{J}_{t} M_{\vartheta, m} \widetilde{\omega}_{V S}^{2}\right)
\end{array}
$$


The analytical solution for the standard undamped Mathieu equation can be obtained through any approximate perturbation method, e.g. multiple scales. Hence, the so-called Ince-Strutt diagram can be defined, representing the stable and unstable regions in the $\delta, \epsilon$ plane (Fig. 9).

It should be remarked that, although this diagram usually refers to truly parametrically excited system (i.e. where the excitation is provided by an external source), it also applies to the investigated 2:1 internal resonance which indeed provides an auto-parametric phenomenon, by which the motion of the driving (hanging) mode is coupled with the motion of the driven (twisting) mode, giving rise to the parametric resonance above the activation threshold.

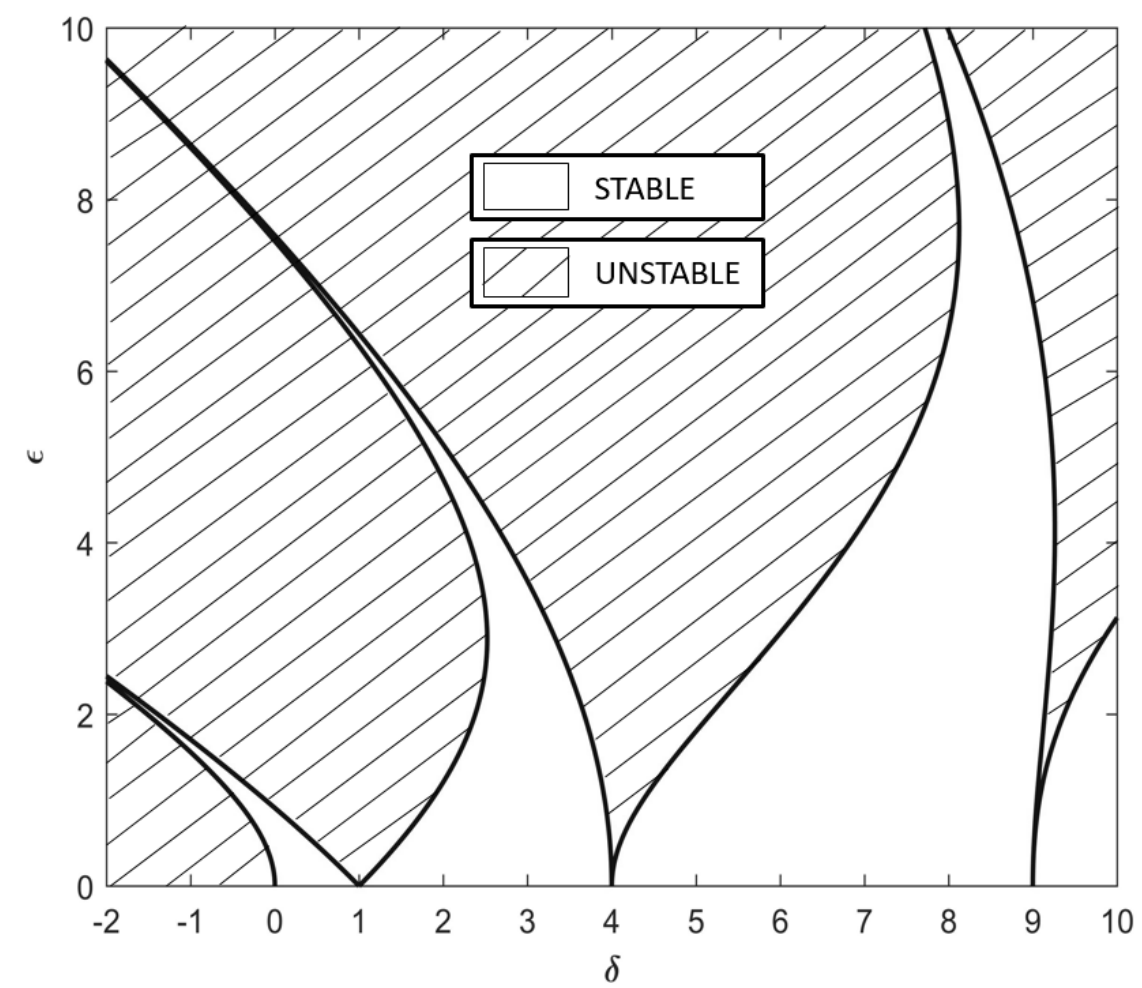

Figure 9: Stable and unstable (shaded) regions in the $\boldsymbol{\delta}, \boldsymbol{\epsilon}$ plane for the undamped Mathieu equation.

According to the diagram, the system shows critical conditions in correspondence of particular ratios between the torsional and the flexural perturbation frequencies, that is $\widetilde{\Omega}_{\vartheta} / \widetilde{\omega}_{V S}=n / 2$. By increasing the internal resonance ratio order $n$, the unstable regions become narrow. Hence the most critical case is $\delta=1$, representing the so-called 2:1 internal resonance condition: the frequency of the flexural perturbation is twice the torsional one. Here the unstable region covers the widest range in correspondence of the lowest values for $\epsilon$, associated to the flexural perturbation amplitude. The main advantage of this approach is that, being based on a standard diagram, it simply requires to know the structural parameters and the expected flexural amplitude in order to assess the stability of the structural system. The main disadvantage is that, being the flexural and the torsional perturbed equation completely decoupled, it does not allow us to take directly into account the aeroelastic effects, which may strongly couple the linear response of the system.

\subsection{Floquet stability analysis of the perturbed equations}

The stability of the complete perturbation system is hereby analyzed by exploiting the Floquet Theorem. 


\subsubsection{Formulation of Floquet stability conditions}

Systems of differential equations of second order with periodic coefficients can be written as the product between a periodic function $P(t)$ and an exponential one $M(t)$ [30]. A solution is asymptotically stable in Lyapunov sense if the periodic function does not diverge. In order to check this property, we need first to reduce the perturbed system (41) of Hill's equation to an equivalent system of the first order.

$$
\dot{x}=J \cdot x
$$

The procedure requires a simple change of variables that leads to the following definition of Jacobian matrix $J$.

$$
\begin{aligned}
& x=\left\{\begin{array}{llll}
z_{P, n} & \gamma_{P, m} & \dot{z}_{P, n} & \dot{\gamma}_{P, m}
\end{array}\right\}^{T} \\
& J=\left\{\begin{array}{cc}
0 & I \\
-M^{-1} \cdot K & -M^{-1} \cdot D
\end{array}\right\} \\
& M=\left\{\begin{array}{cc}
M_{w, n} & 0 \\
0 & \tilde{J}_{t} M_{\vartheta, m}
\end{array}\right\} \\
& D=\left\{\begin{array}{cc}
D_{w, n} & D_{w \vartheta, n m} \\
D_{\vartheta w, m n} & D_{\vartheta, m}
\end{array}\right\} \\
& K=\left\{\begin{array}{cc}
K_{w, n}^{(L)} & K_{w \vartheta, n m}^{(L)} \\
K_{\vartheta w, m n}^{(L)} & K_{\vartheta, m}^{(L)}+K_{\vartheta w, m n}^{(Q)} \cdot z_{n, 0} \cdot \sin \left(\widetilde{\omega}_{V S} \cdot \tau-\varphi\right)
\end{array}\right\}
\end{aligned}
$$

The so-called monodromy matrix measures the variation of the solution vector after a period. In order to compute such a matrix, it is necessary to perform a numerical integration of the equivalent first order system over the reference-forcing period $\widetilde{T}=2 \pi / \widetilde{\omega}_{V S}$ assuming a number of initial conditions equal to the number of unknowns. The initial values have to be chosen in such a way that the fundamental matrix of solutions is unitary on the main diagonal at the initial instant. This allows us to define the monodromy matrix as follows.

$$
C=\left\{[x(\tilde{T})]_{Z_{P, n}(0)=1} \quad[x(\tilde{T})]_{\gamma_{P, m}(0)=1} \quad[x(\tilde{T})]_{\dot{Z}_{P, n}(0)=1} \quad[x(\tilde{T})]_{\dot{\gamma}_{P, m}(0)=1}\right\}
$$

The monodromy matrix is written starting from the solution vector $x$ obtained by numerical time integration of the Hill's equations. To this purpose, the $4^{\circ}$ order Runge-Kutta scheme is implemented. Each column of matrix $C$ contains the solution vector computed after one period $\tilde{T}$ of the parametric excitation. For each column a different initial condition is taken, so that the only non null term is indicated in the subscript and it is unitary.

Finally, stability is granted if none of the absolute values of monodromy matrix' eigenvalues exceeds the unity.

\subsubsection{Stability maps analysis}

In the following, we will comment the numerical results obtained from a parametric analysis of the stability of the perturbed system with airfoil shaped section. Results will be summarized trough the stability maps depending upon circular frequency of vortex shedding $\omega_{V S}$ and mean wind speed level $U$. The stability maps are achieved by selecting a discrete set of values for the two parameters, in order to cover a wide range of frequency, on one hand, and the whole set of wind speed beneath the torsional divergence, on the other hand. For each combination of the 
two parameters, Floquet stability of the system is inspected by the computation of the monodromy matrix (52). If one of the eigenvalues of the monodromy matrix is larger than unity, the system is unstable and a black dot is depicted in the frequency-wind speed plane

Two models will be analyzed. The first one, called Structural, accounts for geometrical and mechanical properties of the suspension bridge only. This preliminary analysis is useful in order to check if the non-linearities of the dynamic system are strong enough to produce internal resonance conditions. In the second one, called Aeroelastic, both structural and aerodynamic effects will be considered to find out if, and under which conditions, wind effect coupled with parametric resonance is able to lead the structure to unstable conditions. Results are of relevance only if the structure reaches those critical conditions in correspondence of a wind speed lower than the flutter one.

Relevant resonance conditions are:

$$
\begin{aligned}
& \text { harmonic : } \widetilde{\omega}_{V S}=\widetilde{\omega}_{i} \\
& \text { subharmonic : } \widetilde{\omega}_{V S}=2 \cdot \widetilde{\omega}_{i} \\
& \text { superharmonic : } \widetilde{\omega}_{V S}=\widetilde{\omega}_{i} / 2 \\
& \text { combinational sum : } \widetilde{\omega}_{V S}=\widetilde{\omega}_{w}+\widetilde{\omega}_{\vartheta} \\
& \text { anti-resonance : } \widetilde{\omega}_{V S}=\left|\widetilde{\omega}_{w}-\widetilde{\omega}_{\vartheta}\right|
\end{aligned}
$$

Since we want to catch just the critical conditions near the classical primary linear resonance, we are mainly interested to the second type $(2: 1)$.

The academic model that is analyzed in the present section has the following structural properties: span $l=1400 \mathrm{~m}$; half-width of the deck $b=15 \mathrm{~m}$; sag of the cable $f=170 \mathrm{~m}$; flexural inertia $m_{d}+2 m_{c}=3.0 \mathrm{e}+04 \mathrm{~kg} / \mathrm{m}$; torsional inertia $J_{t}=5.0 \mathrm{e}+06 \mathrm{kgm}$; flexural stiffness $E_{d} I_{d}=$ $3.8 \mathrm{e}+11 \mathrm{Nm}^{2}$; torsional stiffness $G_{d} J_{d}=1.1 \mathrm{e}+11 \mathrm{Nm}^{2}$; cable stiffness $E_{c} A_{c}=2.4 \mathrm{e}+10 \mathrm{~N}$. Warping stiffness is assumed to be negligible. As a consequence, the dimensionless parameters read: $\chi^{2}=\infty ; \lambda_{1}^{2}=100 ; \mu^{2}=0.5 \% ; \beta^{2}=1$. Let's analyze some numerical results starting from the Structural model. As we can see in Fig. 13, the Structural model is able to catch the main resonances of the system. In fact, besides the primary linear flexural one, the system undergoes resonance also as the forcing frequency is near the torsional one $\left(\widetilde{\omega}_{V S} / \widetilde{\Omega}_{\vartheta, 1}=1\right)$. Since the excitation frequency is associated to flexural motion, this kind of phenomenon can be associated to an internal resonance of kind 1:1 (i.e. harmonic internal resonance). Further, the model is able to catch also the so-called 2:1 internal subharmonic resonance as the flexural motion is characterized by a frequency that doubles the torsional one $\left(\widetilde{\omega}_{V S} / \widetilde{\Omega}_{\vartheta, 1}=2\right)$. On the other hand, the effect of other kinds of resonances is less evident. It is worth noting that the presence of internal resonances reduces drastically the critical wind speed level that, in the case of the simple structural model, coincides with the torsional divergence speed. 


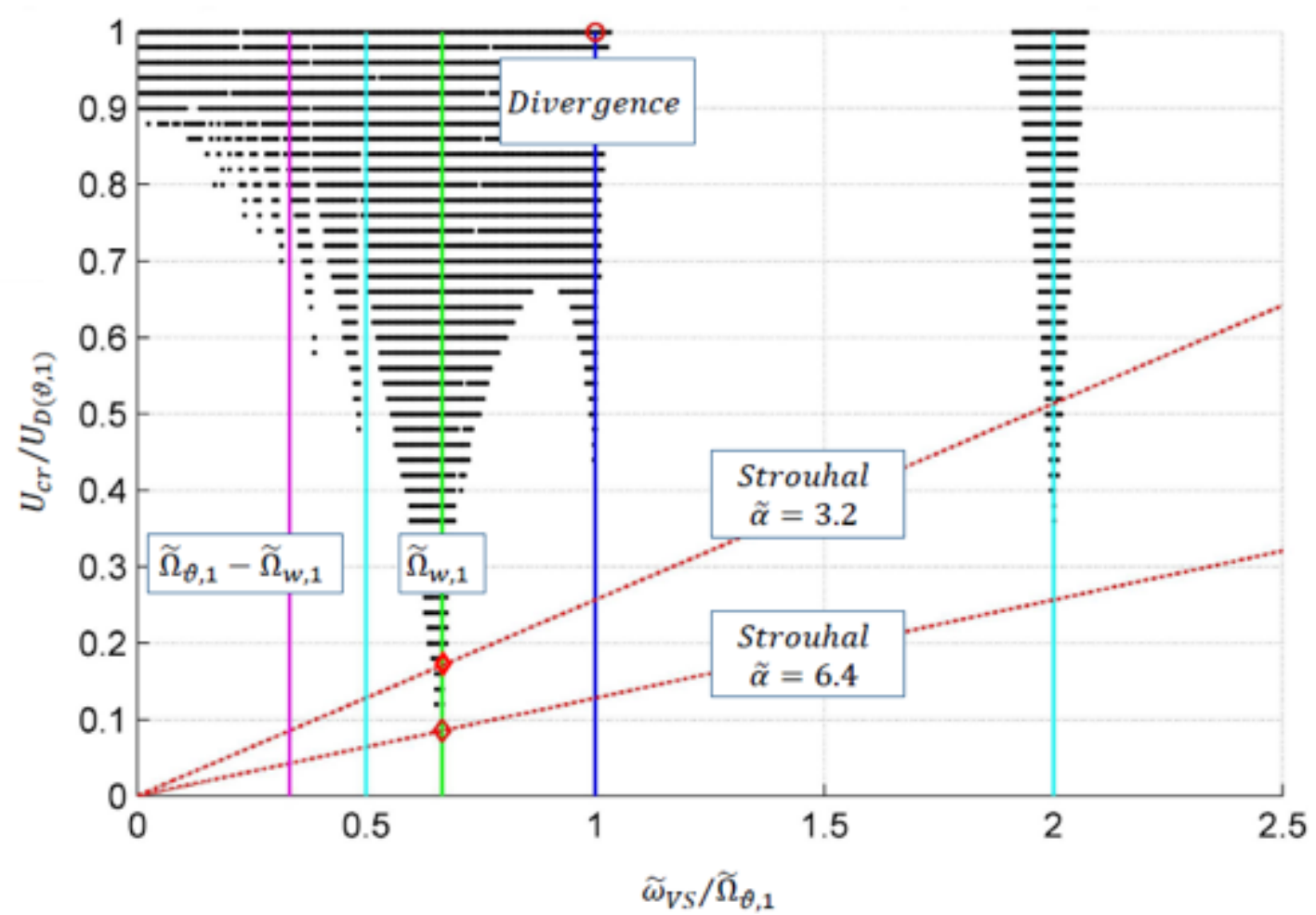

Figure 10: Stability map of Airfoil Structural model ( $1^{\circ}$ flexural and $1^{\circ}$ torsional symmetric modes). $\left[\tilde{J}_{t}=0.6 ; \widetilde{m}_{a}=0.04 ; 2 \tilde{b}=0.18 ; S t=0.1 ; \Delta_{w, 1}=\Delta_{\vartheta, 1}=0.5 \% ; \chi^{2}=\infty ; \lambda_{1}^{2}=100 ; \mu^{2}=0.5 \% ; \beta^{2}=1\right]$.

As already mentioned, in the definition of the dimensionless Strouhal circular frequency $\widetilde{\omega}_{V S}$, a relevant role is played by the deck sectional aspect ratio $\tilde{\alpha}$, as it strongly modifies the slope of the Strouhal curve, which consequently can enter the unstable regions. As we can see in Fig. 10 , by halving the aspect ratio down to $\tilde{\alpha}=3.2$ the vortex-shedding phenomenon is able to explain the main resonant unstable conditions (1:1 flexural and 2:1 torsional resonances).

Hence, we can conclude that very bluff deck sections can be useful to increase torsional and (most importantly) flexural stiffness of the suspension bridge, but they lead the structure to be more susceptible to parametric instabilities due to vortex shedding.

Let's now examine the Aeroelastic model accounting for the fluid-structure interaction effects on the dynamic stability of the system. As mentioned earlier, the fluid-structure interaction makes the frequencies being dependent on the actual wind speed level; consequently, in Fig. 11 we see curved lines representing the main internal or external resonance conditions. Beyond Flutter wind speed threshold any forcing frequency leads the system to unstable conditions. On the contrary, below this critical condition, the effect of parametric resonance is relevant only in correspondence of some critical frequency. It is evident that the Aeroelastic model is less susceptible of Parametric resonance than the Structural one. This is due mainly by the fact that wind-structure effects make the eigen-frequency of the system dependent on the actual wind speed level and, below Flutter onset, they introduce additional damping contributions stabilizing both the flexural and torsional response, Fig. 12. 


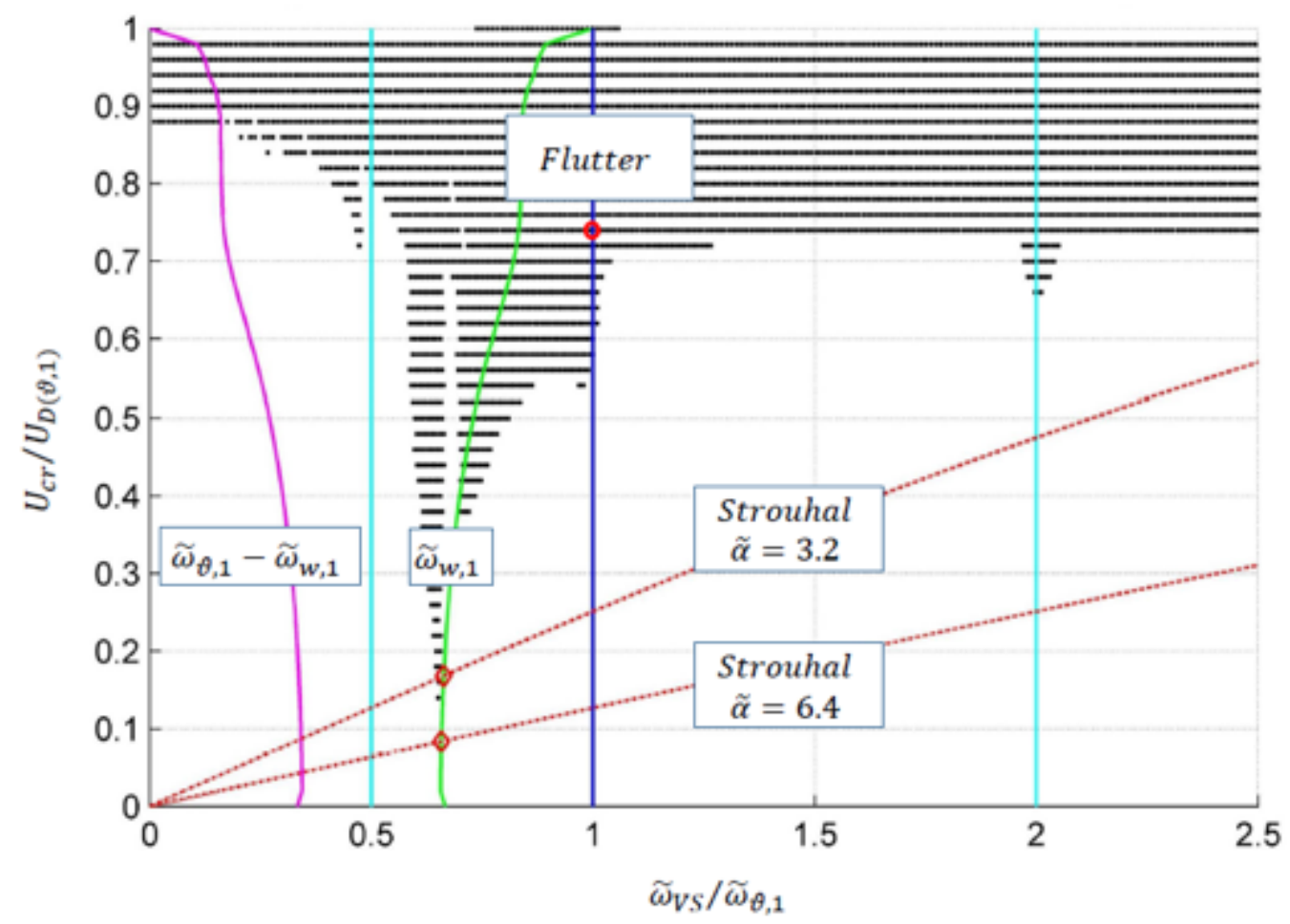

Figure 11: Stability map of Airfoil Aeroelastic model ( $1^{\circ}$ flexural and $1^{\circ}$ torsional symmetric modes). $\left[\tilde{J}_{t}=0.6 ; \tilde{m}_{a}=0.04 ; 2 \tilde{b}=0.18 ; S t=0.1 ; \Delta_{w, 1}=\Delta_{\vartheta, 1}=0.5 \% ; \chi^{2}=\infty ; \lambda_{1}^{2}=100 ; \mu^{2}=0.5 \% ; \beta^{2}=1\right]$.

The previous statement that Flutter onset makes unstable conditions independent from the forcing frequency is not valid everywhere. Indeed, near $\widetilde{\omega}_{V S}=\left(\widetilde{\omega}_{\vartheta, 1}-\widetilde{\omega}_{w, 1}\right)$ we get a large stable region even for wind speed level higher than the Flutter one. In fact, as reported also in different papers [48], the so called anti-parametric resonance with respect to the parametric and additive parametric ones has a stabilizing effect on the dynamic response of the system. This is due primary to the fact that this phenomenon is able to increase significantly the damping of the system. This particular property can be exploited trying to find the optimal geometrical and mechanical parameters able to maximize this intrinsic damping effect.

The physical explanation of this feature hides behind the fact that, as parametric excitation occurs, there is energy transfer between the two main interacting modes. Consequently, since higher order modes are characterized by higher damping ratios, then the system is able to dissipate faster that energy with respect to the system without Parametric excitation that the classical Flutter theory is not able to capture. Notice that we are dealing with an energetic exchange. Hence, being the energy transfer not unidirectional, we should observe modulated vibrations time histories. 


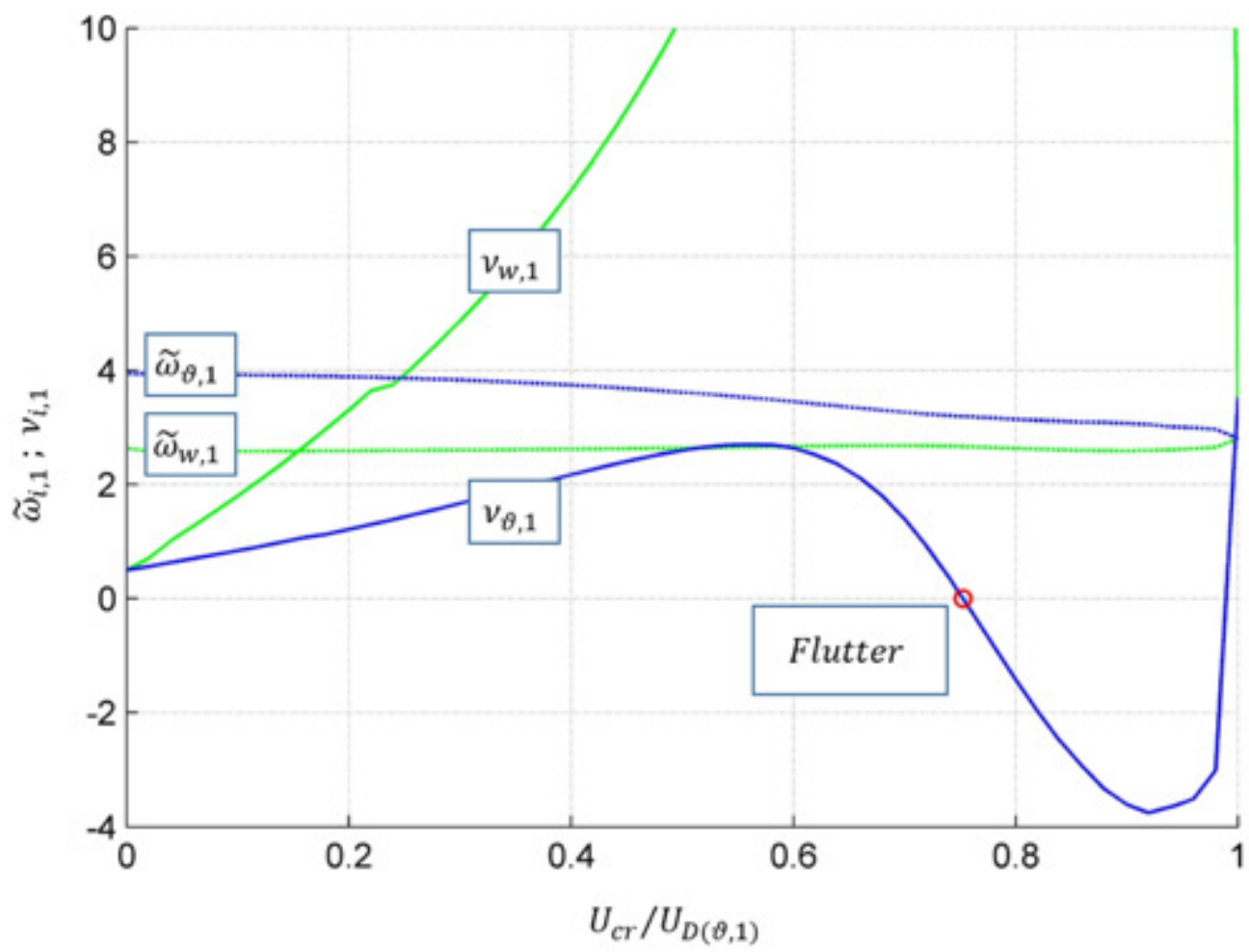

Figure 12: Flutter analysis of Airfoil model ( $1^{\circ}$ flexural and $1^{\circ}$ torsional symmetric modes). $\left[\tilde{J}_{t}=0.6 ; \Delta_{w, 1}=\Delta_{\vartheta, 1}=0.5 \% ; \chi^{2}=\infty ; \lambda_{1}^{2}=100 ; \mu^{2}=0.5 \% ; \beta^{2}=1\right]$.

Now we are able to comment the previous information in terms of flexural antinodal displacements for the single degree of freedom oscillator formulation. In fact, once we define the wind speed and the shedding frequency, we know respectively the forcing term $\Gamma_{0}$ and the complex dynamic amplification factor $H\left(\widetilde{\omega}_{V S}\right)$. Considering Figs. 13-14, we recognize immediately the usual shape characterizing every SDOF forced oscillator. We must remind that the equivalent oscillator is much more damped in the Aeroelastic model than in the Structural one at least below the Flutter onset. 


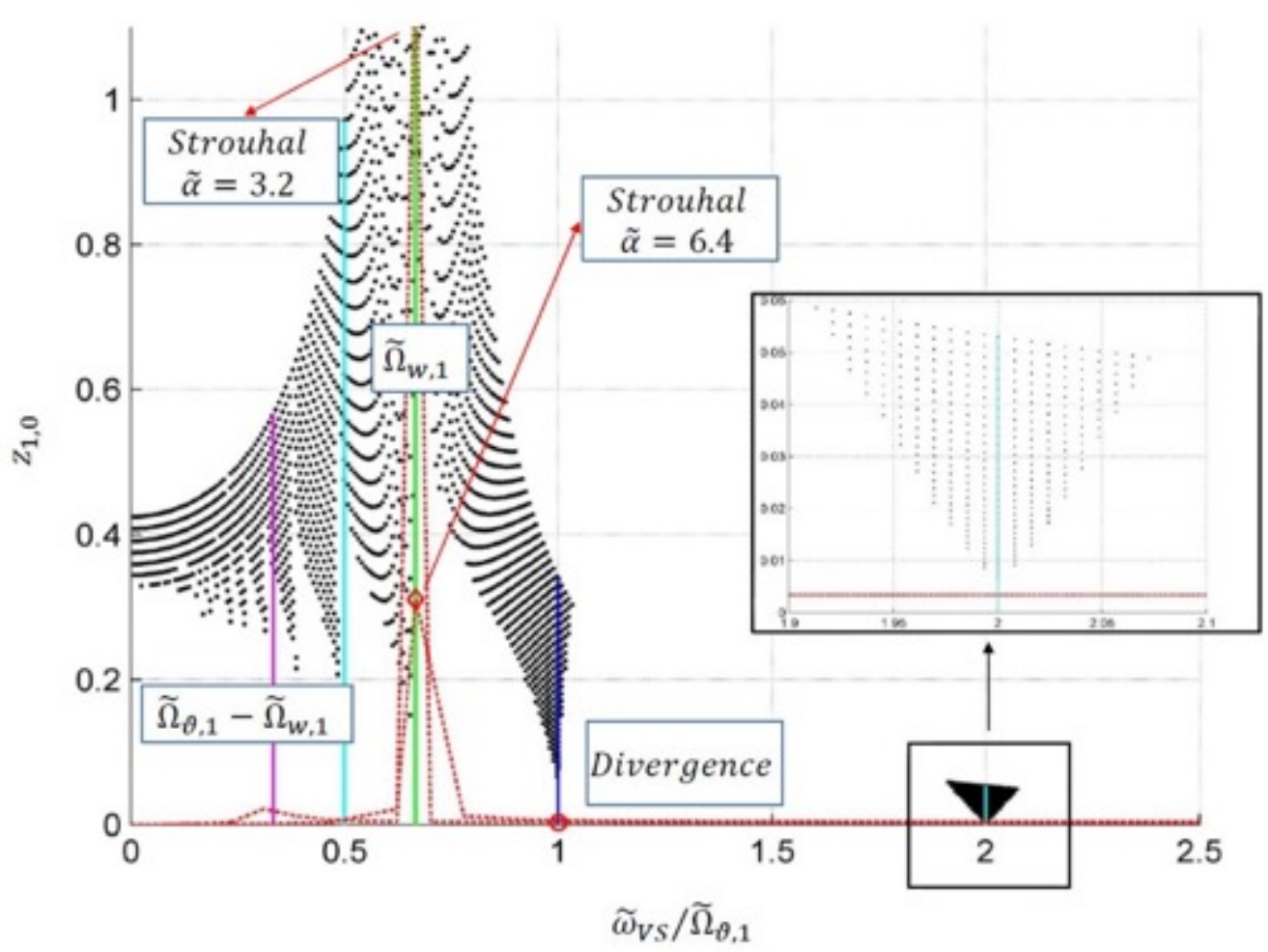

Figure 13: Unstable amplitudes of Airfoil Structural model ( $1^{\circ}$ flexural and $1^{\circ}$ torsional symmetric modes). $\left[\tilde{J}_{t}=0.6 ; \widetilde{m}_{a}=0.04 ; 2 \tilde{b}=0.18 ; S t=0.1 ; \Delta_{w, 1}=\Delta_{\vartheta, 1}=0.5 \% ; \chi^{2}=\infty ; \lambda_{1}^{2}=100 ; \mu^{2}=0.5 \% ; \beta^{2}=1\right]$.

According to Figs. 13-14, it seems that the 2:1 internal resonance is the most critical condition requiring the lowest amplitudes to be activated. Conversely, all the other situations require too much high antinodal displacements that are difficult to observe in real life situations. Indeed, the critical amplitudes in the neighborhood of $2: 1$ resonance are small enough to fulfill approximately the empirical limit threshold $\widetilde{w}_{d, \max }=0.2 \cdot D / f \cong 2 \%$ reminding us that vortex-shedding is a self-limiting phenomenon. 


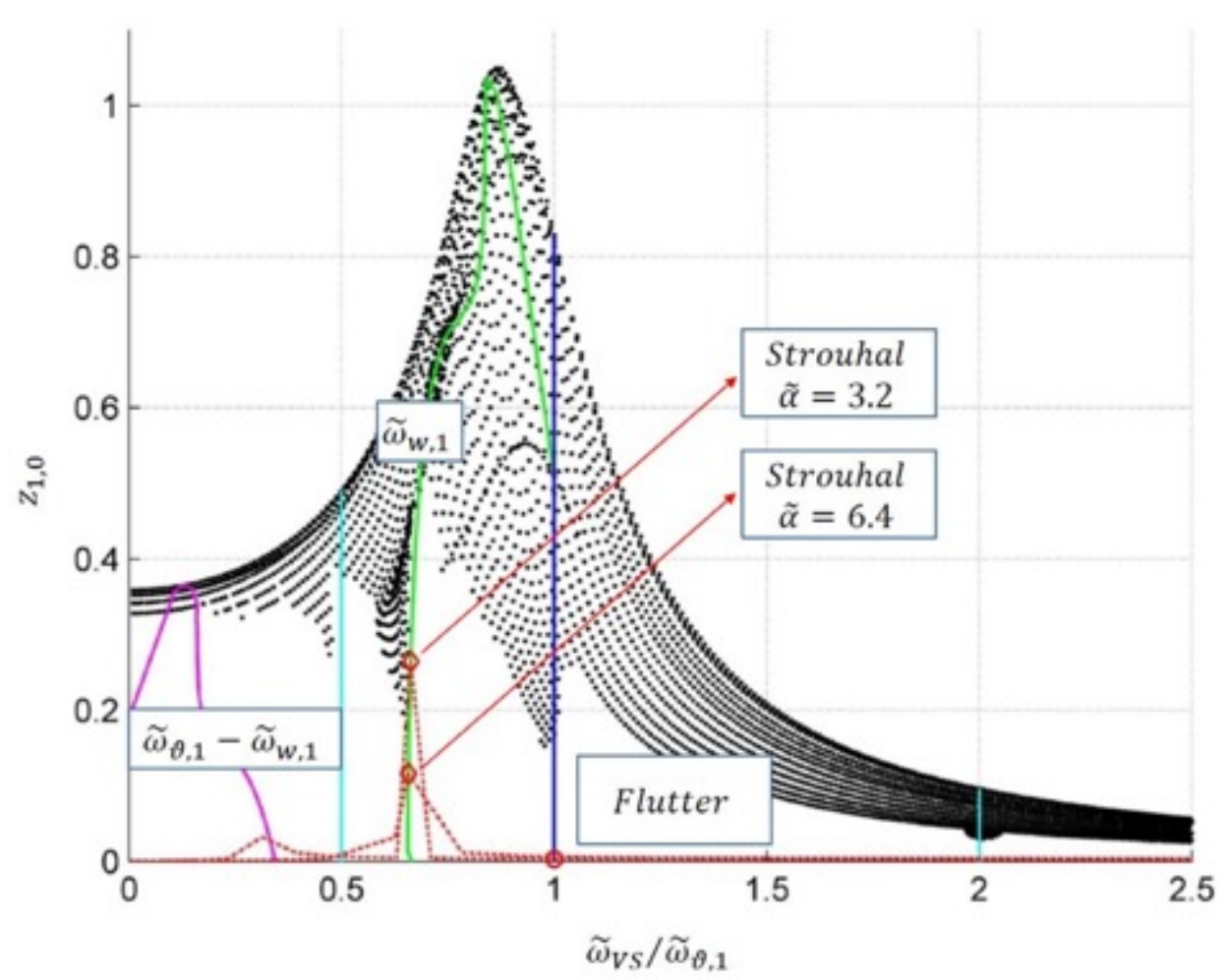

Figure 14: Unstable amplitudes of Airfoil Aeroelastic model ( $1^{\circ}$ flexural and $1^{\circ}$ torsional symmetric modes). $\left[\tilde{J}_{t}=0.6 ; \widetilde{m}_{a}=0.04 ; 2 \tilde{b}=0.18 ; S t=0.1 ; \Delta_{w, 1}=\Delta_{\vartheta, 1}=0.5 \% ; \chi^{2}=\infty ; \lambda_{1}^{2}=100 ; \mu^{2}=0.5 \% ; \beta^{2}=1\right]$.

\subsubsection{Stability maps in Mathieu-type format}

In order to build up a stability map with the same format adopted in the analyses of Mathieu equations, we simply plot the unstable regions in the $\delta, \epsilon$ plane, where the two parameters have been defined in (45). Notice that the definition of $\delta$ given in (45) is valid only for the Structural model but not for the Aeroelastic one for which holds the more general $\delta=4\left(K_{\vartheta, 1}^{(L)} / \tilde{J}_{t} M_{\vartheta, 1}\right) / \widetilde{\omega}_{V S}^{2}$, being the torsional eigen-frequency dependent on the wind speed level. For sake of simplicity we have represented just the stability maps with any reference to the Strohual law or the different sub- or super-harmonic resonance.

The stability map of the Structural model, where only parametric resonance occurs, is able to trace the Mathieu diagram. Some regions in the upper part are missing, since the amplitudes of oscillations are dictated by the wind speed level, whose upper bound is represented by the static divergence, and a SDOF oscillator model governs those amplitudes. Hence, not any point on the map can be reached by a real life physical problem. On the other hand, the unstable regions cannot touch the horizontal axis of null amplitudes due to the presence of structural damping. Though not so much visible, in Fig. 15 we can recognize the dangerous 2:1 parametric resonance condition in correspondence of $\delta=1$.

Concerning the Aeroelastic model, the stability map of Fig. 16 changes very much its shape, though the main features do not. Indeed, the parabolic shaped lines represent unstable conditions associated to wind speed level beyond Flutter onset. Hence, for any values of forcing frequency we get an unstable condition according to Floquet Theory. However, it is still evident that the wind-structure interaction increases the stability of the system beneath the Flutter onset whilst the parametric effects enlarge the unstable regions near the so-called 1:1 and 2:1 resonances as in the classical Mathieu map. 


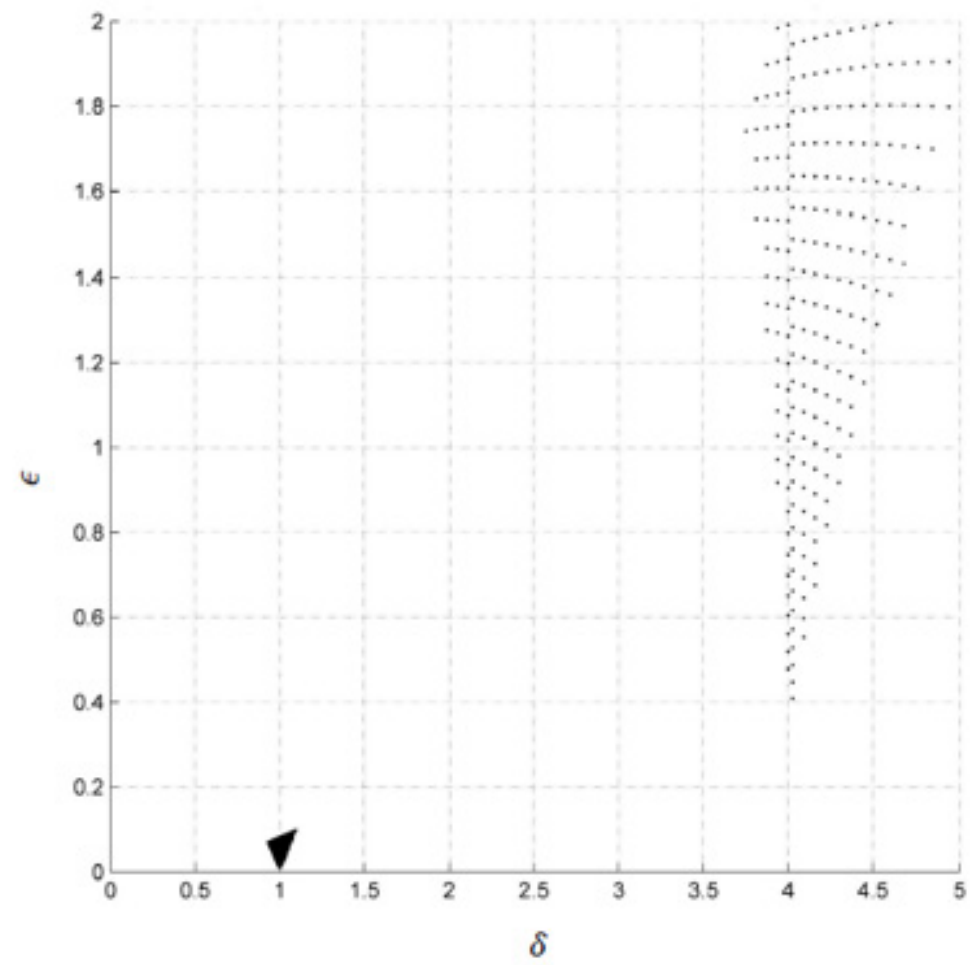

Figure 15: Stability map of Airfoil Structural model ( $1^{\circ}$ flexural and $1^{\circ}$ torsional symmetric modes). $\left[\tilde{J}_{t}=0.6 ; \widetilde{m}_{a}=0.04 ; 2 \tilde{b}=0.18 ; \Delta_{w, 1}=\Delta_{\vartheta, 1}=0.5 \% ; \chi^{2}=\infty ; \lambda_{1}^{2}=100 ; \mu^{2}=0.5 \% ; \beta^{2}=1\right]$.

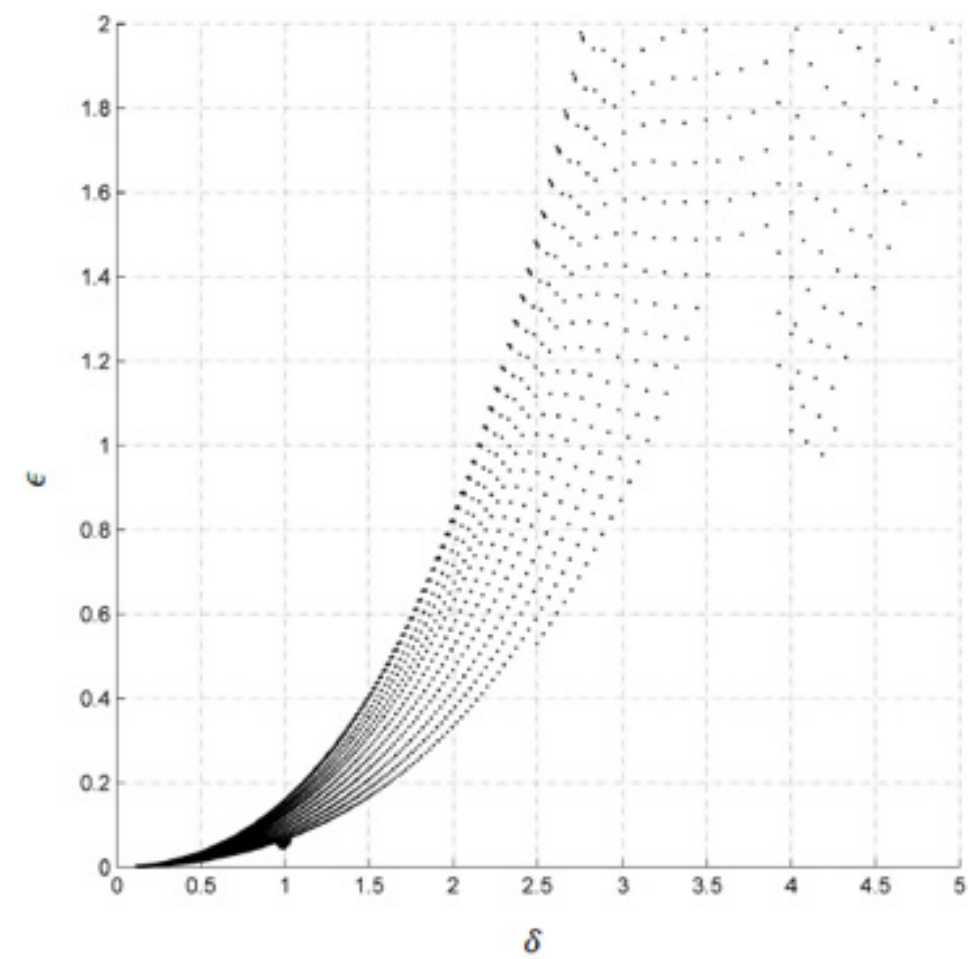

Figure 16: Stability map of Airfoil Aeroelastic model ( $1^{\circ}$ flexural and $1^{\circ}$ torsional symmetric modes). $\left[\tilde{J}_{t}=0.6 ; \widetilde{m}_{a}=0.04 ; 2 \tilde{b}=0.18 ; \Delta_{w, 1}=\Delta_{\vartheta, 1}=0.5 \% ; \chi^{2}=\infty ; \lambda_{1}^{2}=100 ; \mu^{2}=0.5 \% ; \beta^{2}=1\right]$. 


\section{A NUMERICAL APPLICATION TO TNB}

The collapse of the central span of the original Tacoma Narrows suspension bridge (TNB) on November $7^{\text {th }}, 1940$, has been studied in many papers. The first one is the thorough report (the "Carmody Report"), written by a governmental committee consisting of O.H. Ammann, T. Von Karman, and G.B. Woodruff in 1941 [13]. Clark Eldridge, a bridge engineer for the Washington State Toll Bridge Authority, proposed a design in 1938. The central span was $853.4 \mathrm{~m}$ long and $11.9 \mathrm{~m}$ wide, with two lanes. A truss below the roadway was $7.6 \mathrm{~m}$ deep to stiffen the deck against vertical, lateral and torsional displacements. The bridge was too costly, and therefore the Public Works Administration (PWA) later hired a well-known consultant, Leon Moisseiff of New York. He replaced the truss in Eldridge's design with two vertical (stiffening) silicon-steel plate girders along the sides, extending $1.22 \mathrm{~m}$ above and below the roadway. Stringers and laterals with a chevron $(\mathrm{K})$ configuration were placed below the deck. The four lanes deck was $11.9 \mathrm{~m}$ wide and $2.44 \mathrm{~m}$ deep, with a stiffening truss below the roadway and with three sets of diagonal shock absorbers on each side at midspan.

The bridge was opened on July $1^{\text {st }}$, 1940, and since the opening day vertical oscillations appeared due to lateral winds whose speed reached more than $22 \mathrm{~m} / \mathrm{s}$. In those cases, the amplitude ranged from $0.4 \mathrm{~m}$ up to $0.76 \mathrm{~m}$ [28]. Those vertical oscillations were not considered dangerous and they died away due damping forces when the velocity of wind dropped down. On 7 November 1940 the wind speed of $19 \mathrm{~m} / \mathrm{s}$ was measured. The motion of the deck before $10 \mathrm{a} . \mathrm{m}$. was vertical with an amplitude not more than $0.5 \mathrm{~m}$. The frequency of the motion was 36-38 cycles per minute, which was significantly higher than previously measured frequencies. Around 10 a.m. the motion of the central span switched into a torsional mode with a single node at the midspan. The torsional oscillations appeared after the loosening of the midspan cable band on one main cable. The initial frequency was 14 cycles per minute, but after a short time it decreased to 12 cycles per minute, perhaps due to some damage within the deck. The motion of the central span changed form during the subsequent hour, but it was primarily a one-nodetorsional oscillation. The maximum twist angle was about $35^{\circ}$ and the corresponding maximum vertical amplitude was about $4.3 \mathrm{~m}$. The bridge collapsed at 11:10 a.m..

From the parameters of the original Tacoma bridge [44] it follows that the hangers were sufficiently stiff to be considered inextensible in tension and completely slack in compression. Since the moment of collapse, many theories have been presented about the reasons which led to the collapse. The influence of aerodynamic forces has been intensively studied together with some nonlinear phenomena connected with the construction of suspension bridges, namely the nonlinearity of cable systems.

In the present work, we want to consider not only the nonlinearities introduced by the cable system and the ones coming from wind action, but also the effects of parametric flexural excitation of the bridge. From the available data of the structure [44] we can define first the fundamental dimensionless parameters entering in the governing equation of motion.

$$
\lambda_{1}^{2}=168.3 ; \chi^{2}=0.3 ; \mu^{2}=3.8 \cdot 10^{-4} ; \beta^{2}=1.2 \cdot 10^{-4}
$$

With these values we, are able to completely define the eigen-properties of the bridge. The ones of interest are the circular frequencies associated to the fifth symmetric flexural mode and to the first skew-symmetric torsional one. In fact, just before collapse the bridge oscillated according to a dominant flexural motion characterized by 8 internal nodes that is associated to a fifth order symmetric mode; when the snap occurred, torsional oscillations with a central node appeared. 
By means of the definition (9) given for the dimensionless circular eigen-frequency, we are able to define the frequencies associated to the modes of interest.

$$
f_{w, 5}=0.681 \mathrm{~Hz} ; f_{\vartheta, 1}=0.194 \mathrm{~Hz}
$$

By comparing the numerical results with those observed during the collapse $[13,28,51,52]$, we can state that the flexural frequency just computed is higher than the measured one (of about $0.617 \mathrm{~Hz}$ ) whilst the computed torsional frequency is slightly lower than the one observed (about $0.2 \mathrm{~Hz}$ ). The reason hides behind some assumption we made. On one hand, the model neglects the deformability coming from the motion of pylons, assumed to be perfectly rigid, and the one coming from the hangers, for which a tenso-rigid constitutive model applies, making the response stiffer. On the other hand, the assumption of perfectly hinged ends gets rid of the flexural stiffening contribution coming from cables' section and from side spans, making the response softer. Hence we can conclude that flexural response is mostly affected by the first group of assumptions whilst torsional motion by the second one.

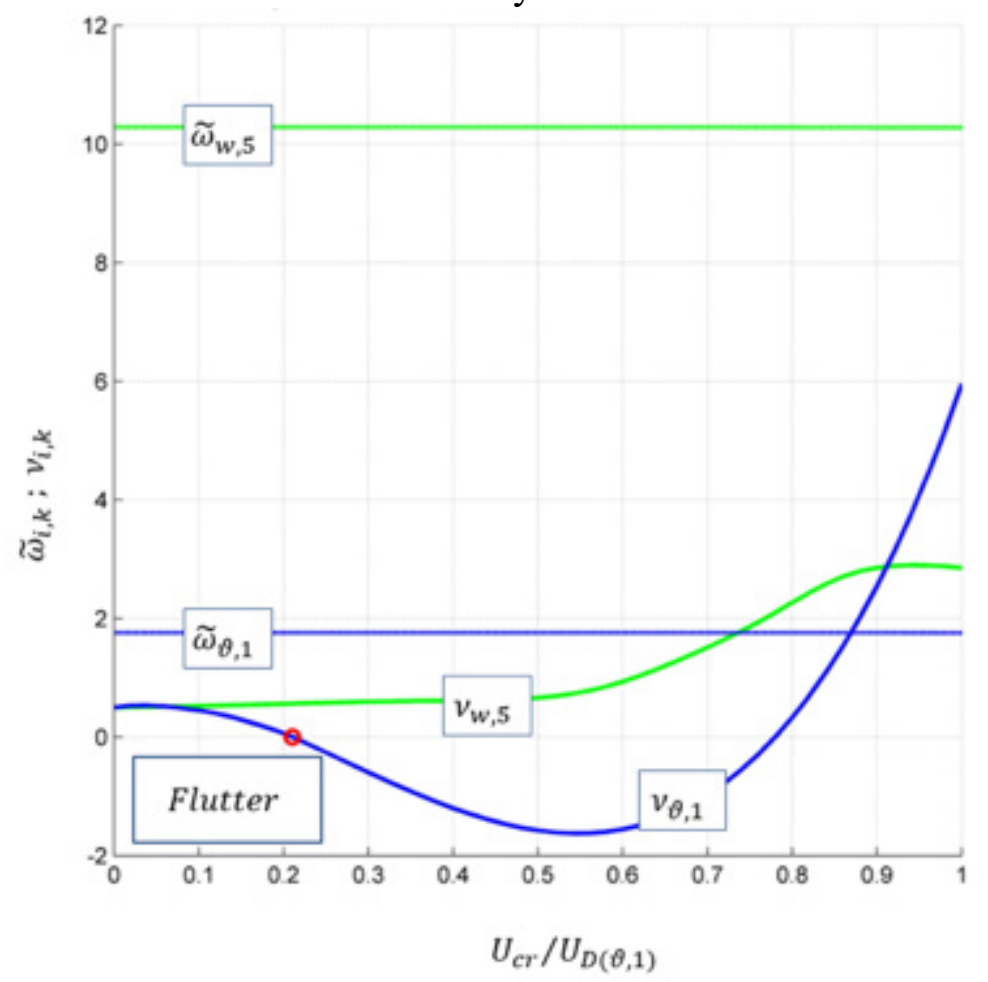

Figure 17: Flutter analysis of TNB ( $5^{\circ}$ flexural symmetric and $1^{\circ}$ torsional skew-symmetric modes). $\left[\tilde{U}_{t}=0.47 ; \widetilde{m}_{a}=0.014 ; 2 \tilde{b}=0.168 ; S t=0.1 ; \Delta_{w, 5}=\Delta_{\vartheta, 1}=0.5 \% ; \chi^{2}=0.3 ; \lambda_{1}^{2}=168.3 ; \mu^{2}=0.38 \% 0 ;\right.$ $\left.\beta^{2}=0.12 \% 0\right]$.

Further, we estimate the critical wind speed level $U_{F}$ that is able to lead the structure to Flutter onset, making use of the Flutter Derivatives proper of the TNB and no more the ones of the Airfoil. Scanlan, in his paper [52], argues that the TNB suffers of a pure torsional Flutter being the flexural to torsional coupling very weak. Hence, looking for the lowest critical wind speed, we should perform the numerical analysis focusing on the interaction between the torsional mode of lowest order and any flexural one. We found that the lower bound is represented by the $1^{\circ}$ skew-symmetric torsional mode, and we arbitrarily choose the $5^{\circ}$ symmetric for the flexural one (see Fig.17), thus obtaining 


$$
U_{F}=8.8 \mathrm{~m} / \mathrm{s}
$$

This result slightly overestimates those $\left(U_{F}=8.3 \mathrm{~m} / \mathrm{s}\right)$ recently obtained by Scanlan [51] and the experimental ones $\left(U_{F}=7.1 \mathrm{~m} / \mathrm{s}\right)$ by Farquhardson [52] in 1954 on a full model 1:50 scale dynamic wind tunnel test.

Finally, we can focus on the critical conditions analyzing the stability map associated to the aeroelastic model for the TNB. In order to compare numerical results with those measured during the collapse we need to take in consideration the $5^{\circ}$ order symmetric flexural mode, that characterized the structure just before the sudden snap condition, and the $1^{\text {st }}$ order skew-symmetric torsional one, that led the structure to collapse.

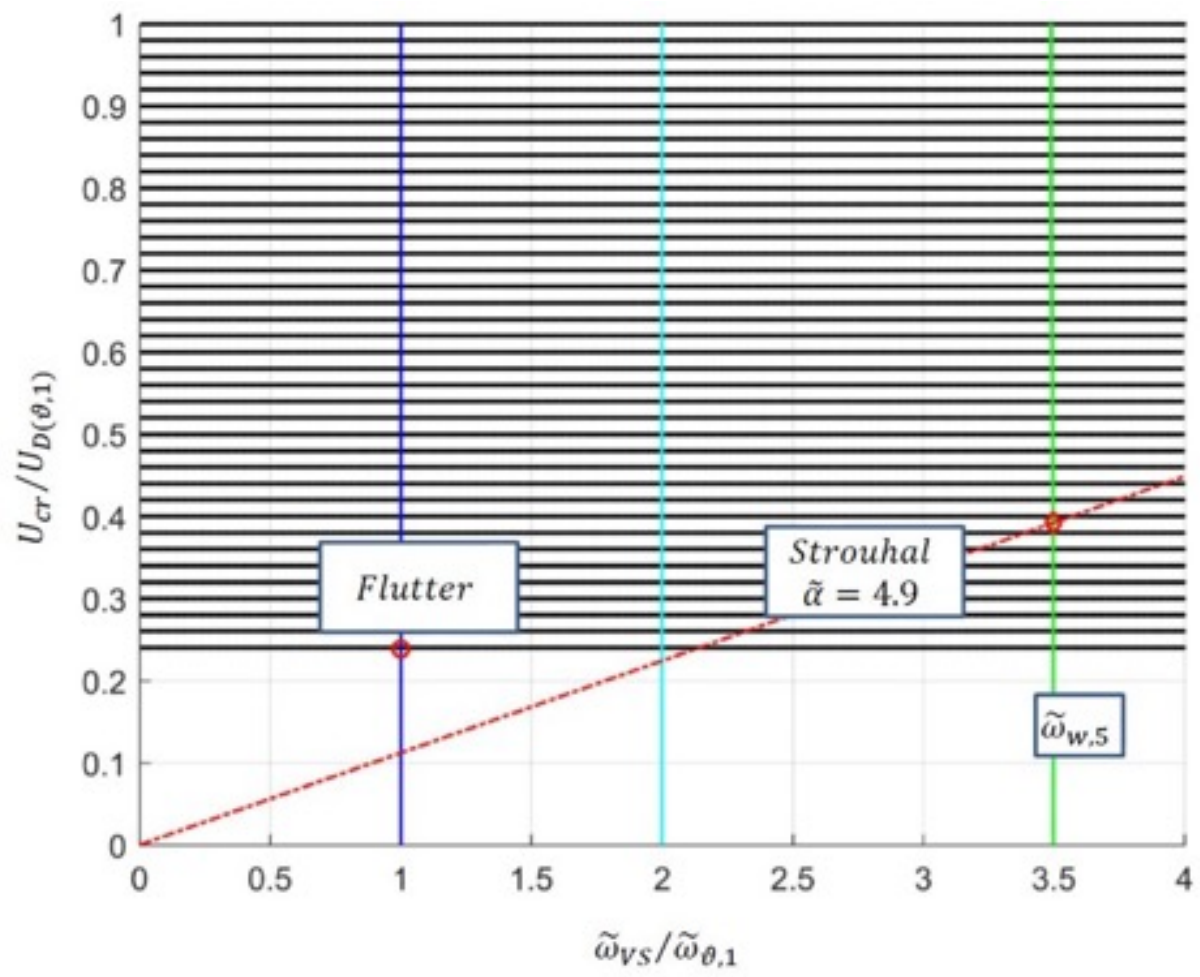

Figure 18: Stability map of TNB Aeroelastic model ( $5^{\circ}$ flexural symmetric and $1^{\circ}$ torsional skew-symmetric). $\left[\tilde{J}_{t}=0.47 ; \tilde{m}_{a}=0.014 ; 2 \tilde{b}=0.168 ; S t=0.1 ; \Delta_{w, 5}=\Delta_{\vartheta, 1}=0.5 \% ; \chi^{2}=0.3 ; \lambda_{1}^{2}=168.3 ; \mu^{2}=0.38 \%\right.$; $\beta^{2}=0.12 \%$ ].

We have not reported the figure related to Structural model since the absence of aeroelastic effects preclude the possibility of unstable conditions in the wind speed range below the static divergence limit.

The Strouhal model for vortex shedding predicts lock-in condition, with the $5^{\circ}$ symmetric flexural mode, as the wind speed velocity reaches approximately $17 \mathrm{~m} / \mathrm{s}$. This is coherent with what was observed before the collapse, being the reported wind speed around $19 \mathrm{~m} / \mathrm{s}$. Indeed, at this wind speed level, the vortex shedding frequency reaches approximately $0.74-0.83 \mathrm{~Hz}$ (being St $=0.1 \div 0.11$ and $\mathrm{D}=2.44 \mathrm{~m}$ ). That value falls in the lock-in range, i.e. $0.8-1.3$ times the 5-th mode flexural frequency $(0.68 \mathrm{~Hz})$.

However, from Fig. 18 it seems that the stability threshold is dictated by the Flutter onset rather than Parametric resonance. Indeed, the critical wind speed remains constant for any value of 
perturbation frequency. This is correct as long as the perturbation amplitudes $z_{n, 0}$ are governed by the response of a SDOF oscillator forced by vortex shedding (as explained in Sec 5.1.2). In order to focus on the loss of stability due to parametric resonance, we may fix the wind speed level slightly under the Flutter onset $\left(\tilde{u}=\tilde{u}_{F}-0.02\right)$, where the vortex shedding lock-in with the 2:1 torsional resonance. Then, the amplitude of the perturbation $z_{5,0}$, defined in (7) as the modal amplitude normalized with respect to the initial cable sag, is increased arbitrarily, i.e. irrespective of its connection with the wind speed.

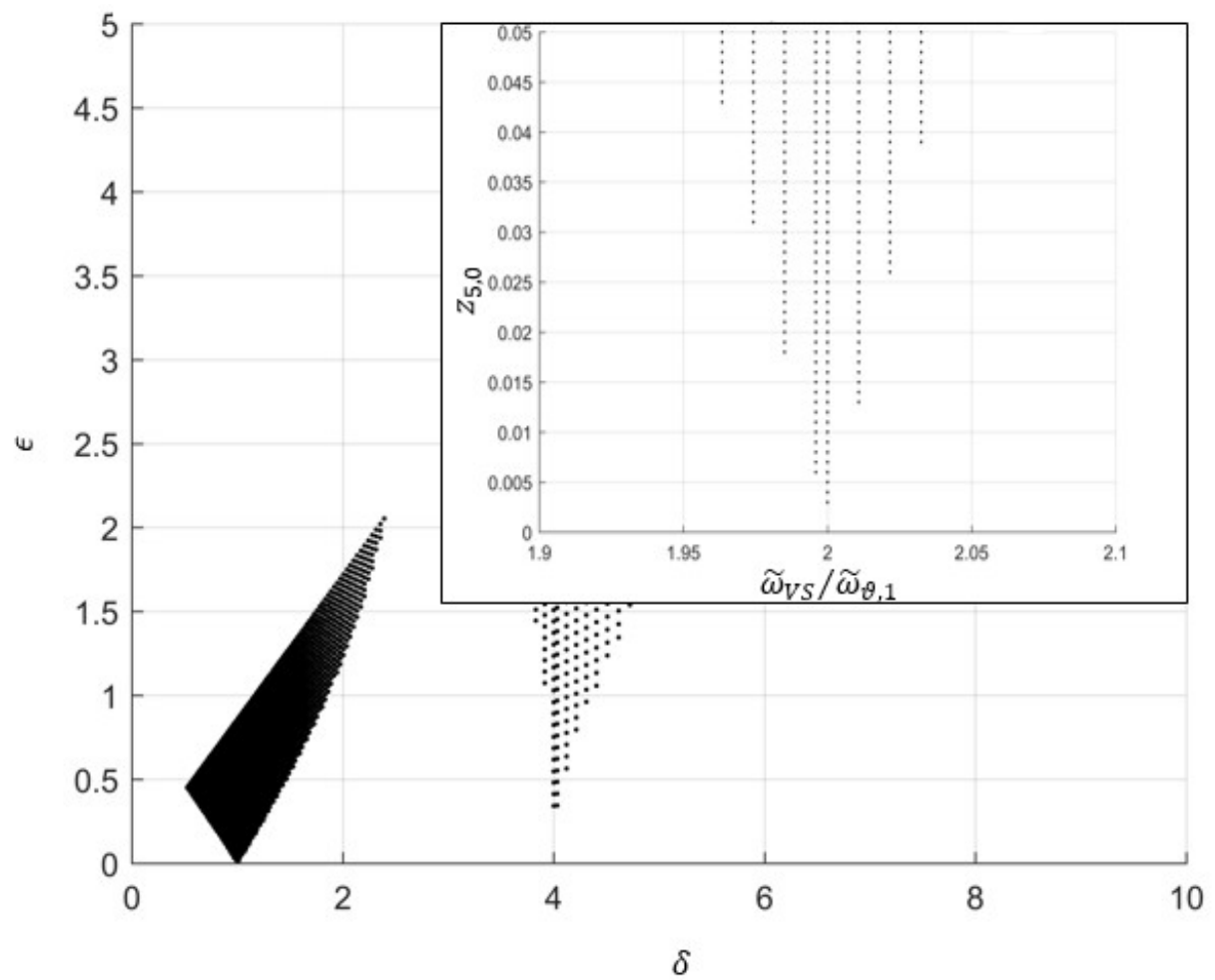

Figure 19: Stability map of TNB Aeroelastic model ( $5^{\circ}$ flexural symmetric and $1^{\circ}$ torsional skew-symmetric). $\left[\tilde{J}_{t}=0.47 ; \tilde{m}_{a}=0.014 ; 2 \tilde{b}=0.168 ; S t=0.1 ; \Delta_{w, 5}=\Delta_{\vartheta, 1}=0.5 \% ; \chi^{2}=0.3 ; \lambda_{1}^{2}=168.3 ; \mu^{2}=0.38 \%\right.$; $\beta^{2}=0.12 \%$ ].

Such hypothesis allow us to trace the stability map in the $\delta, \epsilon$ plane (Fig. 19), with the classical pattern of the Mathieu damped equation. The inset in Fig. 19 is referred to the plane $z_{5,0}, \widetilde{\omega}_{V S} / \widetilde{\omega}_{\vartheta, 1}$ and points out the lowest critical amplitudes: the tongue around the 2:1 resonance is the least affected by damping effects. We can estimate that the critical modal displacement is about $0.22 \mathrm{~m}$. Hence, vortex shedding seems to be able to activate internal parametric $2: 1$ resonance with the $1^{\circ}$ skew-symmetric torsional mode as the flexural amplitudes of the $5^{\circ}$ symmetric one reaches $0.20-0.30 \mathrm{~m}$, being impossible to get perfectly a $2: 1$ lock-in condition. A validation of the proposed approach can be obtained by considering the time response of the system in correspondence of a perturbation that is (i) within and (ii) just beyond the stability threshold. The original nonlinear system of equations (12-13-19-20) is now considered. For the investigation of the 2:1 parametric resonance, we make a modal projection taking into account just the interaction between the $5^{\circ}$ symmetric flexural mode and the $1^{\circ}$ skew-symmetric torsional one. The numerical integration has been performed by means of a classical Runge-Kutta integration scheme. The forcing term is represented by vortex shedding, eq. (26), with an exci- 
tation frequency that is twice the torsional eigenfrequency. As initial conditions, a small torsional perturbation is introduced (equal to $1 \%$ in dimensionless format), whereas the flexural displacement starts from naught. First, we consider that the vortex shedding excitation is not high enough to bring the flexural parametric perturbation inside the unstable region. More precisely, the induced vibration is characterized by a dimensionless modal amplitude 0.0022 , that is just within the stability threshold depicted in the inset in Fig. 19. The time response in such a situation is represented in Fig. 20: the response is stable because the flexural DOF oscillates according to the forcing term and the torsional response is damped in time. In the second analysis, the magnitude of the forcing term is slightly increased, so that the dimensionless modal amplitude is 0.0032 (just beyond the stability threshold). The time response, Fig. 21, shows clearly an unstable behavior, since the torsional DOF diverges in time.

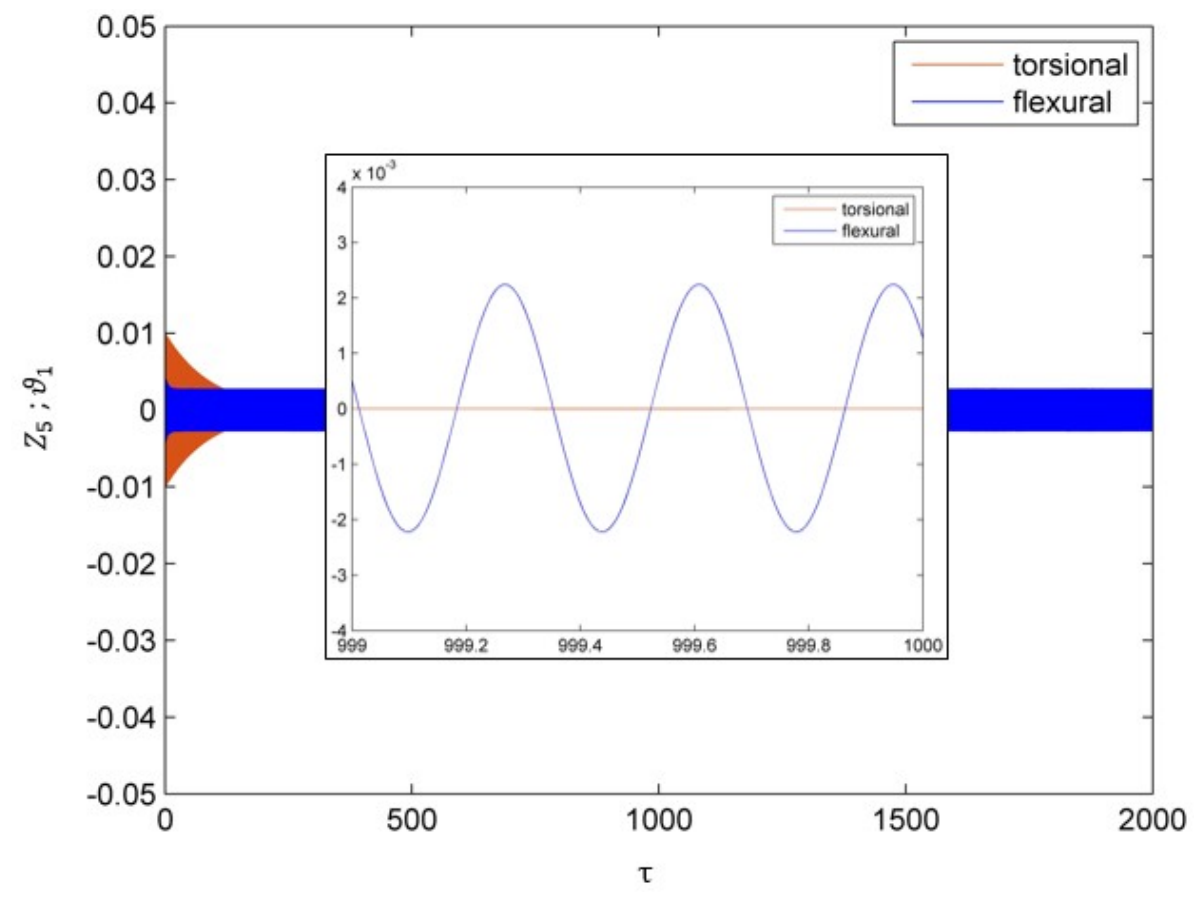

Figure 20: Stable time response of TNB Aeroelastic model ( $5^{\circ}$ flexural sym and $1^{\circ}$ torsional skew-sym). $\left[\tilde{J}_{t}=0.47 ; \tilde{m}_{a}=0.014 ; 2 \tilde{b}=0.168 ; S t=0.1 ; \Delta_{w, 5}=\Delta_{\vartheta, 1}=0.5 \% ; \chi^{2}=0.3 ; \lambda_{1}^{2}=168.3 ; \mu^{2}=0.38 \%\right.$; $\beta^{2}=0.12 \%$ ]. 


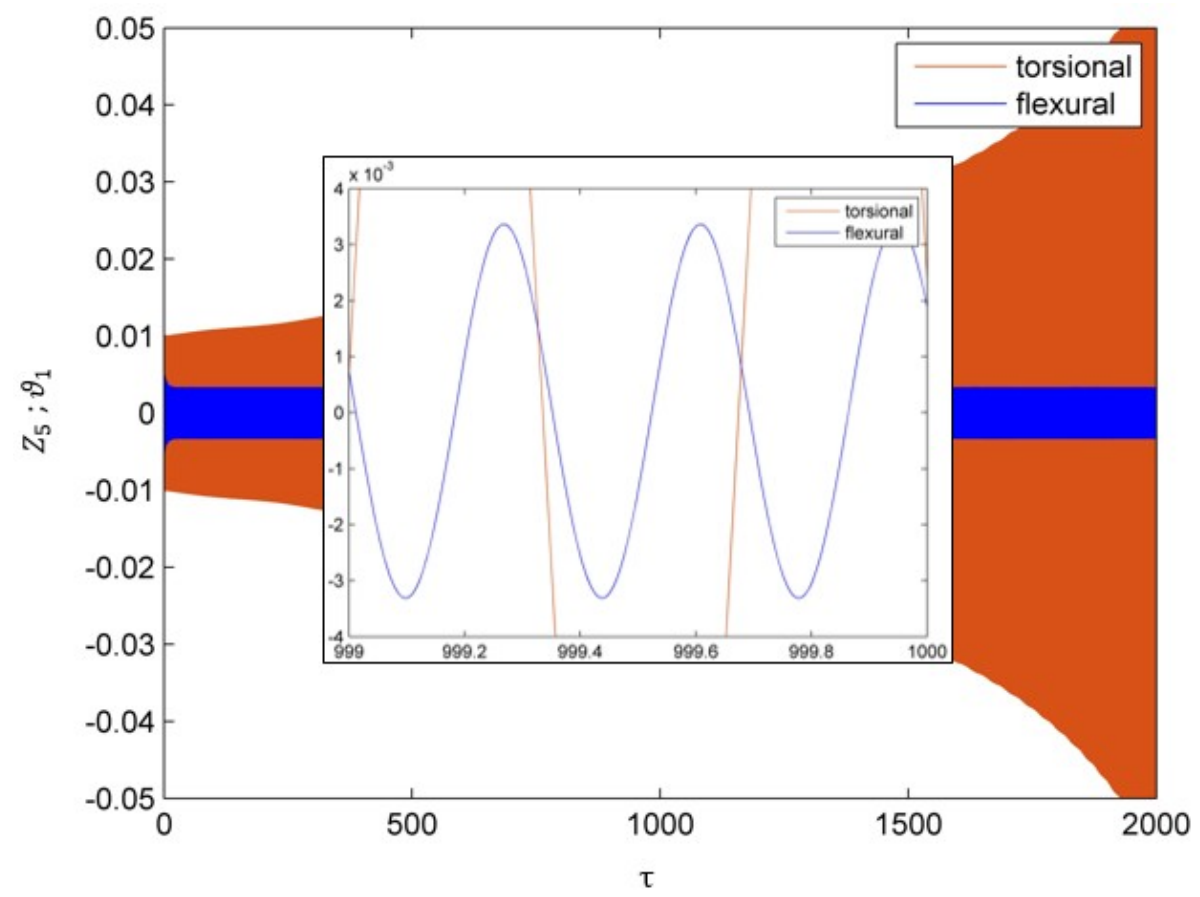

Figure 21: Unstable time response of TNB Aeroelastic model ( $5^{\circ}$ flexural sym and $1^{\circ}$ torsional skew-sym). $\tilde{J}_{t}=0.47 ; \widetilde{m}_{a}=0.014 ; 2 \tilde{b}=0.168 ; S t=0.1 ; \Delta_{w, 5}=\Delta_{\vartheta, 1}=0.5 \% ; \chi^{2}=0.3 ; \lambda_{1}^{2}=168.3 ; \mu^{2}=0.38 \%$; $\beta^{2}=0.12 \%$ ].

We return now to the Aeroelastic model, where perturbation amplitudes are governed by vortex shedding. We focus on the vertical antinode displacements necessary for the onset of unstable conditions, see Fig. 21. In correspondence of the lock-in point with $\widetilde{\omega}_{w, 5}$, the unstable antinode displacement is about $0.69 \mathrm{~m}$. This is an important data since allows us to state that the hangers are taut, being the slackening threshold (computed with the procedure summarized in Sec. 2.2) for the fifth order flexural symmetric mode equal to $0.73 \mathrm{~m}$. That confirms the validity of the whole procedure. Again, concerning the comparison with real phenomenon, we notice that in effect, during collapse of the TNB, in the central span no hanger seems to slack. Furthermore, just before the bridge motion snaps to the dominant torsional motion, vertical oscillations of about $0.50-0.70 \mathrm{~m}$ were observed, showing satisfactory agreement with the values we just determined.

Finally, we can interpret the result saying that, as the structure was excited by resonant vortex shedding, it vibrated steadily according to the fifth flexural symmetric mode. In view of the flexural amplitudes of $0.73 \mathrm{~m}$, the response could easily (due to low amplitude threshold $0.22 \mathrm{~m}$ ) diverge in time by an internal parametric resonance with the first order skew-symmetric torsional mode. The possible rupture of a hanger can explain the frequency shift of the flexural motion to the 2:1 resonance with the torsional one and the failure of the bridge. 


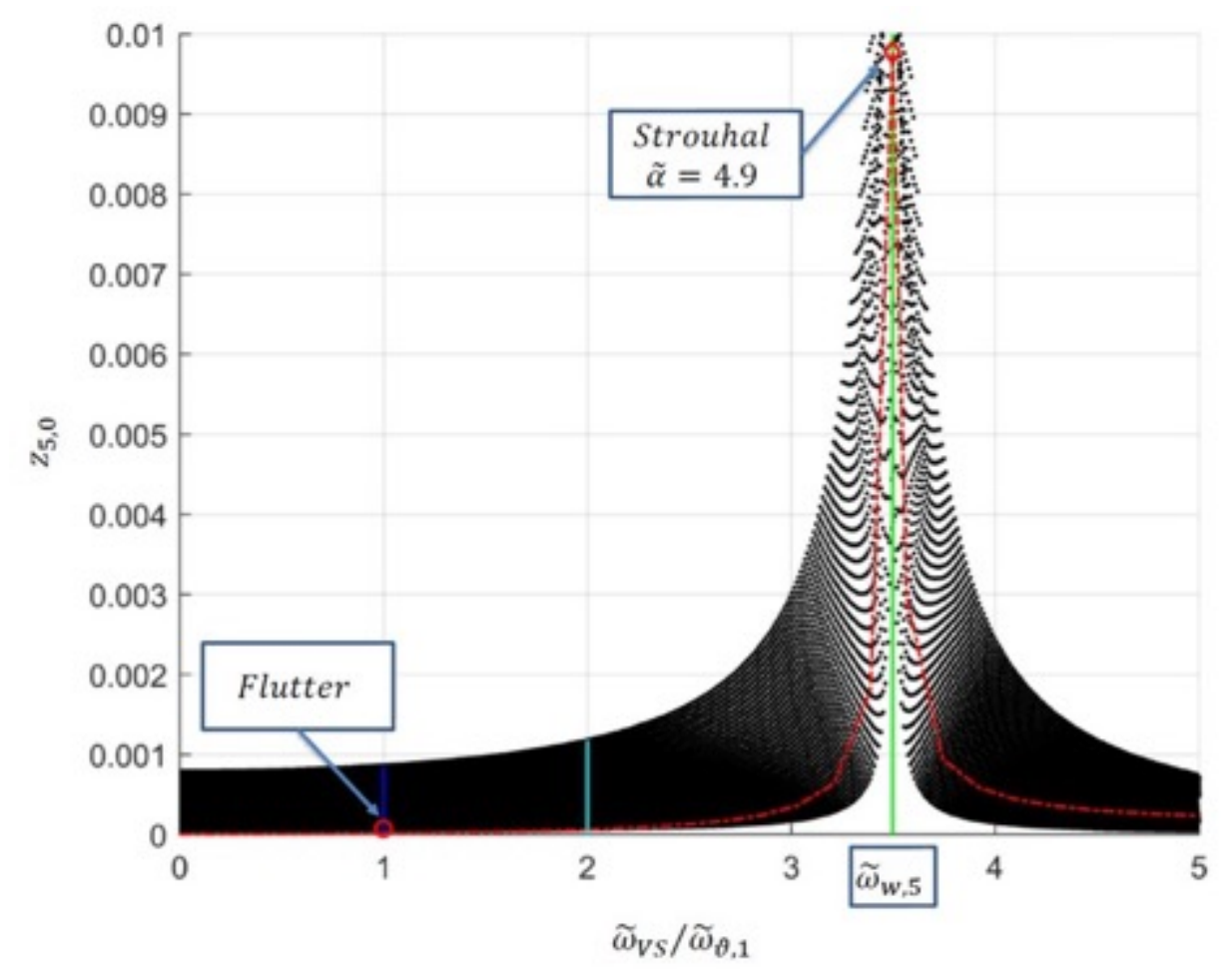

Figure 22: Unstable amplitudes of TNB Aeroelastic model ( $5^{\circ}$ flexural sym and $1^{\circ}$ torsional skew-sym). $\left[\tilde{J}_{t}=0.47 ; \tilde{m}_{a}=0.014 ; 2 \tilde{b}=0.168 ; S t=0.1 ; \Delta_{w, 5}=\Delta_{\vartheta, 1}=0.5 \% ; \chi^{2}=0.3 ; \lambda_{1}^{2}=168.3 ; \mu^{2}=0.38 \%\right.$; $\left.\beta^{2}=0.12 \% 0\right]$.

\section{CONCLUSIONS}

In this paper, a non-linear dynamic model of a suspension bridge is devised, with the purpose of providing a unified framework for the study of aeroelastic and internal parametric resonance instabilities.

Following the classical Deflection Theory, it has been possible to write the nonlinear static flexural response of a suspension bridge. The next step has consisted in the generalization of the displacement field in order to account for the torsional response of the deck-cables system. A deep insight in analytical aerodynamics paves the way for the study of aeroelastic effects. In view of the frequency dependence of the aeroelastic derivatives, wind forces introduce additional mass, damping and stiffness that not only couples the linear equations of motion but also lead the system to be no more self-adjoint. In fact, it loses its symmetries both in damping and stiffness matrices, making the structure susceptible respectively to flutter instabilities and static divergence problems.

The non-linear system has been treated in the framework of Floquet stability theory, thus achieving the stability maps in terms of wind speed and frequency of vortex shedding. After a thorough examination of the achieved results, we can conclude saying that parametric resonance is a critical phenomenon that can be activated by vortex-shedding as far as the sectional shape factor is low enough. This should warn very much engineers, since a phenomenon like vortex-shedding is usually taken in consideration just concerning serviceability limit states. On the contrary, we have just found that under certain conditions such a phenomenon may lead to very strong and critical unstable conditions in correspondence of wind speed that can be considered safe with respect to classical static divergence or dynamic flutter instabilities. 
The present study can be improved accounting for the possible slackening of vertical hangers directly in the code written to construct the stability maps, which may introduce an additional non-linearity to the system. In fact, the structural model considered herein is based on perfectly bilateral behavior of hangers, that seems a reasonable assumption for perturbation analyses, so that the deck displacement parameters are univocally connected to the cable displacements. In the presence of large upward displacement, e.g. for the investigation of the post-critical nonlinear response, slackening may occur, thus leading to a loss of stiffness for the whole system. In spite of this limitation, the model still provides valid results: in fact, in the cases presented the unstable behavior corresponds to displacements that are lower than the ones necessary for slackening initiation. In the future development of this work, when dealing with higher modes in the framework of multi modal approach, it will be unavoidable to introduce the loss of stiffness due to slackening, since as we have observed initiation will be much easily feasible.

\section{REFERENCES}

[1] A. Pugsley, The theory of suspension bridges. 2nd ed. Edward Arnold, 1968.

[2] D.B. Steinman, A practical treatise on suspension bridges. Wiley, 1953.

[3] F. Bleich, C. Mccullogh, R. Rosecrans, G. Vincent, The mathematical theory of vibration in suspension bridges, U.S. Government Printing Office, 1950.

[4] D. B. Steinman, A generalized deflection theory for suspension bridges, Transaction of ASCE, 100, 1133-1234, 1935.

[5] J. Luco, J. Turmo, Linear vertical vibrations of suspension bridges: A review of continuum models and some new results, Soil Dynamics and Earthquake Engineering, 30, 769-781, 2010.

[6] H. Irvine, Cable structures, MITpress, 1981.

[7] A. M. Abdel-Ghaffar, Vertical vibration analysis of suspension bridges, ASCE Journal of Structural Division, 106, 2053-2075, 1980.

[8] A. M. Abdel-Ghaffar, Free torsional vibrations of suspension bridges, ASCE Journal of Structural Division, 105, 767-788, 1979.

[9] A. M. Abdel-Ghaffar, Free lateral vibrations of suspension bridges, ASCE Journal of Structural Division, 104, 503-525, 1978.

[10] A. M. Abdel-Ghaffar, Suspension bridge vibration: continuum formulation, ASCE Journal of the Engineering Mechanics Division, 108, 1215-1232, 1982.

[11] A.M. Abdel-Ghaffar, L. Rubin, Nonlinear free vibrations of suspension bridges: Theory, Journal of Engineering Mechanics, 109, 313-329, 1983.

[12] A.M. Abdel-Ghaffar, L. Rubin, Nonlinear free vibrations of suspension bridges: Application, Journal of Engineering Mechanics, 109, 330-345, 1983.

[13] O.H. Ammann, T. Von Kármán, G.B. Woodruff, The failure of the Tacoma Narrows Bridge : a report to the honorable John M. Carmody, Federal Works Agency, 1941.

[14] R. L. Bisplinghoff, H. Ashley, R. L. Halfman, Aeroelasticity, Addison-Wesley, 1955.

[15] E. Simiu, R.H. Scanlan, Wind effects on structures, Wiley, 1996.

[16] T. Theodorsen, General theory of aerodynamic instability and the mechanism of flutter, NACA Report n. 496, 1979.

[17] L. Salvatori, C. Borri, Frequency- and time-domain methods for the numerical modeling of full-bridge aeroelasticity, Computers and Structures, 85, 675-687, 2007.

[18] A. Arena, W. Lacarbonara, Nonlinear parametric modeling of suspension bridges under aeroelastic forces: torsional divergence and flutter, Nonlinear Dynamics, 70, 2487-2510, 2012 . 
[19] D. Sado, Energy transfer in two-degree-of-freedom vibrating system : a survey, Journal of Theoretical and Applied Mechanics, 31, 151-173, 1993.

[20] A.H. Nayfeh, D.T. Mook, Nonlinear Oscillation, John Wiley \& Sons, 1995.

[21] Y. A. Rossikhin, M. V. Shitikova, Analysis of nonlinear free vibrations of suspension bridges, Journal of Sound and Vibration, 186, 369-393, 1995.

[22] M. Cevik, M. Pakdemirli, Non linear vibrations of suspension bridges with external excitation, International Journal of Nonlinear Mechanics, 40, 901-923, 2005.

[23] A. H. Nayfeh, Introduction to perturbation techniques, Wiley, 1993.

[24] G. Arioli, F. Gazzola, A new mathematical explanation of what triggered the catastrophic torsional mode of the Tacoma Narrows Bridge, Applied Mathematical Modelling, 39, 901912, 2015.

[25] K. S. Moore, Large torsional oscillations in a suspension bridge: Multiple periodic solutions to a nonlinear wave equation, SIAM Journal on Mathematical Analysis, 33, 14111429, 2002.

[26] P.J. McKenna, Large torsional oscillations in suspension bridges revised: Fixing an old approximation, American Mathematics Monthly, 106, 1-18, 1999.

[27] R. Ardito, A. Capsoni, A. Guerrieri, Internal parametric resonance and aeroelastic effects for long span suspension bridges, Proceedings $5^{\text {th }}$ ECCOMAS Conference on Computational Methods in Structural Dynamics and Earthquake Engineering, COMPDYN 2015, Crete, Greece, 25-27 May 2015, M. Papadrakakis, V. Papadopoulos, V. Plevris (eds.).

[28] R.H. Plaut, Snap loads and torsional oscillations of the original Tacoma Narrows Bridge, Journal of Sound and Vibration, 309, 613-636, 2008.

[29] R.H. Plaut, F.M. Davis, Sudden lateral asymmetry and torsional oscillations of section models of suspension bridges, Journal of Sound and Vibration 307, 2007, 894-905, 2007

[30] L. Cesari, Asymptotic behaviour and stability problems in ordinary differential equations, Springer, 1971.

[31] G. Herrmann, W. Hauger, On the interrelation of divergence, flutter and auto-parametric resonance, Ingeneur-Archiv, 42, 81-88, 1973.

[32] L. Bruno, F. Venuti, A. Scotti, Limit of hangers linearity in suspension footbridge dynamics: A new section model, Journal of Sound and Vibration, 330, 6387-6406, 2011.

[33] V. Sepe, G. Augusti, A deformable section model for the dynamics of suspension bridges. Part I: Model a linear response, Wind and Structures, Vol. 4, No. 1, 1-18, 2001.

[34] V. Sepe, M. Diaferio, G. Augusti, A deformable section model for the dynamics of suspension bridges. Part II: Nonlinear analysis and large amplitudes oscillations, Wind and Structures, Vol. 6, No. 6, 451-470, 2003.

[35] J. M. W. Brownjohn, F. Magalhaes, E. Caetano, A. Cunha, Ambient vibration re-testing and operational modal analysis of the Humber Bridge, Engineering Structures, 32, 20032018, 2010.

[36] F. Nieto, S. Hernandez, J. A. Jurado, A. Mosquera, Analytical approach to sensitivity analysis of flutter speed in bridges considering deck mass, Advances in Engineering Software, 42, 117-129, 2011.

[37] S. Adnur, M. Gunaydin, A. C. Altunisik, B. Sevim, Construction stage analysis of Humber suspension bridge, Applied Mathematical Modelling, 36, 5492-5505, 2012.

[38] K. Kaptan, S. Tezacn, S. Altin, S. Cherry, Dynamic analysis of suspension bridges and full scale testing, Procedia Engineering, 14, 1065-1070, 2011.

[39] N. M. Apaydin, Earthquake performance assessment and retrofit investigations of two suspension bridges in Instanbul, Soil Dynamics and Earthquake Engineering, 30, 702-710, 2010. 
[40] F. Ubertini, Effects of cables damage on vertical and torsional eigenproperties of suspension bridges, Journal of Sound and Vibrations, 333, 2404-2421, 2014.

[41] H. Wang, T. Tao, R. Zhou, X. Hua, A. Kareem, Parameter sensitivity study on flutter stability of long-span triple-tower suspension bridge, Journal of Wind Engineering and Industrial Aerodynamics, 128, 12-21, 2014.

[42] D. Cobo del Arco, A. C. Aparicio, Preliminary static analysis of suspension bridges, Engineering Structures, 23, 1096-1103, 2001.

[43] R. Karoumi, Some modelling aspects in the nonlinear finite element analysis of cable supported bridges, Computer and Structures, 71, 397-412, 1999.

[44] J. Malik, Sudden lateral asymmetric and torsional oscillations in the original Tacoma suspension bridge, Journal of Sound and Vibration, 332, 3772-3789, 2013.

[45] H.Y. Farsani, D.T. Valentine, A. Arena, W. Lacarbonara, P. Marzocca, Indicial functions in the aeroelasticity of bridge decks, Journal of Fluids and Structures, 48, 203-215, 2014.

[46] L. Carassale, T. Wu, A. Kareem, Nonlinear aerodynamic and aeroelastic analysis of bridges: Frequency domain approach, Journal of Engineering Mechanics, 140, 04014051, 2014.

[47] A. Arena, W. Lacarbonara, P. Marzocca, Post critical behaviour of suspension bridges under nonlinear aerodynamic loading, Journal of Computational and Nonlinear Dynamics, 11, 011005, 2016.

[48] J. Welte, T. Kniffka, H. Ecker, Parametric excitation in a two degree of freedom MEMS system, Shock and Vibrations, 20, 1113-1124, 2013.

[49] A. Larsen, J. H. Walther, Discrete vortex simulation of flow around five generic bridge deck sections, Journal of Wind Engineering and Industrial Aerodynamics, 77-78, 591-602, 1998.

[50] A. Larsen, Aerodynamics of the Tacoma Narrows Bridge - 60 Years Later, Structural Engineering International, No. 4, 2000.

[51] R. H. Scanlan, E. Simiu, Aeroelasticity of civil engineering structures, in: E. H. Dowell (Ed.), A Modern Course in Aeroelasticity, 4th reviewed and enlarged edition, Kluwer Academic Publishers, 2004.

[52] K.Y. Billah, R.H. Scanlan, Resonance, Tacoma Narrows Bridge failure, and undergraduate physics textbooks, American Journal of Physics, 59, 118-124, 1991. 